\title{
Ampliando a densidade de coletas botânicas na região da bacia hidrográfica do Alto Paranapanema: Caracterização florística da Floresta Estadual e da Estação Ecológica de Paranapanema
}

\author{
Roque Cielo-Filho ${ }^{1}$, João Batista Baitello ${ }^{1,4}$, João Aurélio Pastore ${ }^{1}$, Osny Tadeu de Aguiar ${ }^{1}$, \\ Silvana Cristina Pereira Muniz de Souza ${ }^{1}$, Maria Teresa Zugliani Toniato ${ }^{2}$, \\ Conceição Rodrigues de Lima $^{3} \&$ Adriano Peres Ribeiro ${ }^{I}$ \\ ${ }^{1}$ Herbário Dom Bento Pickel (SPSF), Divisão de Dasonomia, Instituto Florestal, \\ Rua do Horto, 931, CEP 02377-000, São Paulo, SP, Brasil, www.iflorestal.sp.gov.br \\ ${ }^{2}$ Estação Experimental de Bauru, Divisão de Florestas e Estações Experimentais, Instituto Florestal, \\ Av. Rodrigues Alves, 38-25, Bauru, CEP 17030-000, São Paulo, SP, Brasil \\ ${ }^{3}$ Estação Experimental de Tupi, Divisão de Florestas e Estações Experimentais, Instituto Florestal, \\ Rod. Luiz de Queiroz, Km 149, Piracicaba, CEP 13428-000, São Paulo, SP, Brasil \\ ${ }^{4}$ Autor para correspondência: João Batista Baitello, e-mail: baitello@if.sp.gov.br
}

CIELO-FILHO, R., BAITELLO, J.B., PASTORE, J.A., AGUIAR, O.T., SOUZA, S.C.P.M., TONIATO, M.T.Z., LIMA, C.R. \& RIBEIRO, A.P. Increasing the botanical collecting density at the Alto Paranapanema Basin region: Floristic survey of the State Forest and Ecological Station of Paranapanema. Biota Neotrop. 9(3): http://www.biotaneotropica.org.br/v9n3/en/abstract?inventory+bn02009032009.

\begin{abstract}
The need for increasing the botanical collecting effort at the national scale, as to aid in the identification of priority areas for conservation as to improve the knowledge about the national biota, has been highlighted in several recent analyses concerning the Brazilian biodiversity. For the plants, the collecting density at the national scale $\left(0.44\right.$ dried specimens $\left./ \mathrm{km}^{2}\right)$ is well bellow the minimum for a complete knowledge about the flora ( 3 dried specimens $/ \mathrm{km}^{2}$ ) or even for an assessment of the richness of a given region ( 1 dried specimen $/ \mathrm{km}^{2}$ ). Recent analysis revealed that even in the South and Southwest regions, where the infra-structure is better and the number of botanists greater, the collecting density is inadequate. In the São Paulo state, some regions have been considered poorly collected. The Alto Paranapanema basin is one of these regions. This paper presents the results of a botanical inventory at the Paranapanema municipality conservation units (2332' $02^{\prime \prime} \mathrm{S}$ and $48^{\circ} 45^{\prime} 29^{\prime}$ ' W) and is part of a broader project which aims to contribute for an increase in the botanical collecting density at that region. The State Forest and the Ecological Station of Paranapanema have 900 ha of Seasonal Semideciduous Forest. The pre-existing trails and the trails established for this research were visited monthly between June 2007 and July 2008. 693 fertile specimens were collected and 489 species were identified, belonging to 294 genus and 98 families, according to the following distribution: Myrtaceae (37 species), Fabaceae-Faboideae (32), Rubiaceae (28), Bignoniaceae (23), Asteraceae (20), Lauraceae (17), Apocynaceae (15), Melastomataceae (13), Euphorbiaceae, Malvaceae e Fabaceae-Mimosoideae (12 each). Fifteen species are under extinction risk. The distribution of the species among the life forms reveals 291 trees $(59.5 \%), 72$ lianas (14.7\%), 58 shrubs $(11.9 \%)$, 45 herbs (9.2\%), 19 epiphytes $(3.9 \%)$ and 4 hemiparasites $(0.8 \%)$. The collecting density in the municipality of Paranapanema rose from 0.027 to 0.71 dried specimens $/ \mathrm{km}^{2}$.

Keywords: Atlantic forest, Semideciduous forest, biodiversity assessment, collecting effort, life forms.
\end{abstract}

CIELO-FILHO, R., BAITELLO, J.B., PASTORE, J.A., AGUIAR, O.T., SOUZA, S.C.P.M., TONIATO, M.T.Z., LIMA, C.R. \& RIBEIRO, A.P. Ampliando a densidade de coletas botânicas na região da bacia hidrográfica do Alto Paranapanema: Caracterização florística da Floresta Estadual e da Estação Ecológica de Paranapanema. Biota Neotrop. 9(3): http://www.biotaneotropica.org.br/v9n3/pt/abstract?inventory+bn02009 032009.

Resumo: A necessidade de ampliação da densidade de coleta em escala nacional, tanto para subsidiar a identificação de áreas prioritárias para conservação como para ampliar o conhecimento sobre a biota do país, tem sido enfatizada em diversas análises recentes sobre a biodiversidade brasileira. No caso das plantas, a densidade de coleta em escala nacional $\left(0,44\right.$ exsicatas $\left./ \mathrm{km}^{2}\right)$ está bem abaixo do considerado adequado para um conhecimento completo da flora (3 exsicatas $/ \mathrm{km}^{2}$ ) ou mesmo para a avaliação da riqueza de uma determinada região $\left(1\right.$ exsicata $\left./ \mathrm{km}^{2}\right)$. Análises recentes têm revelado que mesmo nas regiões Sul e Sudeste, dotadas de mais infra-estrutura e maior número de botânicos, a densidade de coletas é insatisfatória. No estado de São Paulo algumas regiões têm sido consideradas carentes em esforço de coleta. Uma dessas regiões corresponde à bacia hidrográfica do Alto Paranapanema. Este trabalho apresenta os resultados de um inventário realizado na Floresta Estadual e Estação Ecológica de Paranapanema (2332' $02^{\prime}$ ' S e $48^{\circ} 45^{\prime}$ '29” O), e é parte de um projeto mais 
amplo, cujo objetivo é ampliar a densidade de coletas botânicas naquela região. As unidades de conservação possuem 900 ha de Floresta Estacional Semidecidual. As trilhas pré-existentes e as abertas para este estudo foram georreferenciadas e percorridas mensalmente no período de junho de 2007 a julho de 2008. Foram efetuadas 693 coletas de plantas férteis, sendo inventariadas 489 espécies, pertencentes a 294 gêneros e 98 famílias botânicas, assim distribuídas nas formas de vida: 291 árvores (59,5\%), 72 lianas (14,7\%), 58 arbustos (11,9\%), 45 ervas (9,2\%), 19 epífitas $(3,9 \%)$ e 4 hemiparasitas $(0,8 \%)$. Quinze espécies encontram-se ameaçadas de extinção. A riqueza de espécies concentrou-se em Myrtaceae (37 espécies), Fabaceae-Faboideae (32), Rubiaceae (28), Bignoniaceae (23), Asteraceae (20), Lauraceae (17), Apocynaceae (15), Melastomataceae (13), Euphorbiaceae, Malvaceae e Fabaceae-Mimosoideae (12 cada). A densidade de coleta no município de Paranapanema passou de 0,027 para 0,71 exsicatas $/ \mathrm{km}^{2}$.

Palavras-chave: floresta Atlântica, floresta Semidecidual, biodiversidade, esforço de coleta, formas de vida.

\section{Introdução}

Em 1992 o Brasil assinou a Convenção sobre Diversidade Biológica (CDB), cujo texto foi posteriormente endossado pelo Congresso Nacional através do Decreto Legislativo 02, de 1994 (Brasil 1994). Ao ratificar a CDB o país assumia, perante a comunidade internacional, vários compromissos referentes a temas e questões relativas à biodiversidade, sintetizados na tríade conservação, utilização sustentável e repartição de benefícios. Um desses compromissos diz respeito à identificação dos componentes da diversidade biológica. Neste ponto, as ações preconizadas pela Política Nacional de Meio Ambiente convergem com uma das linhas de pesquisa subsidiárias mais importantes dentro das ciências biológicas, qual seja, a definição de áreas com pouco conhecimento biológico e o inventário da biodiversidade dessas áreas (Lewinsohn \& Prado 2002, Peixoto \& Thomas 2005, Pirani 2005, Kury 2006). Assumindo esse compromisso, o Brasil lançou-se a um tremendo desafio tendo em vista a sua mega-biodiversidade (Mittermeier et al. 1997). Estima-se que a biota brasileira atualmente conhecida seja composta por algo em torno de 200 mil espécies e que o número total de espécies no país, considerando também aquelas atualmente desconhecidas, possa chegar a 2 milhões (Lewinsohn \& Prado 2002). As estimativas para a diversidade de plantas brasileiras variam de acordo com os autores (veja compilação em Shepherd 2003), contudo, análises recentes têm mostrado alguma concordância ao apontar um total de espécies entre 44 e 56 mil (Shepherd 2003, Giulietti et al. 2005, Lewinsohn \& Prado 2002, Peixoto \& Thomas 2005). De acordo com a estimativa de Giulietti et al. (2005) a flora brasileira compreenderia 19\% das plantas do mundo.

Infelizmente, essa enorme diversidade de plantas está pobremente documentada em herbários nacionais, haja vista a baixa densidade de coletas estimada com base nos acervos desses herbários, 0,44 exsicatas $/ \mathrm{km}^{2}$ (Giulietti et al. 2005). Outros autores apresentaram estimativas um pouco distintas para densidade de coletas, contudo, concordaram ao apontar as regiões Sul e Sudeste como as mais coletadas, com densidade de coleta de 1,7 e 2,59 exsicatas $/ \mathrm{km}^{2}$, respectivamente (Peixoto \& Morim 2003). Entretanto, Shepherd (2003) verificou um importante aspecto ao analisar esses dados, chamando a atenção para o fato de que boa parte do material botânico contido nos acervos das regiões Sul e Sudeste provêm de coletas realizadas em outros estados. Segundo o autor, se considerada a proporção de $20 \%$ de coletas locais, em relação ao total de exsicatas depositadas em herbários da região Sudeste, a densidade de coletas de fanerógamas é reduzida de 1,81 para 0,36 exsicatas $/ \mathrm{km}^{2}$. Para o estado de São Paulo, a correção acima resultou em uma redução acentuada, de 2,47 para 0,5 exsicatas $/ \mathrm{km}^{2}$. As informações contidas em Durigan et al. (2008) permitem calcular uma densidade de 0,25 registros georreferenciados de fanerógamas $/ \mathrm{km}^{2}$.

Os esforços de coleta no estado de São Paulo, além de escassos, são também muito mal distribuídos, se concentrando em municípios com atributos particulares como alta diversidade ou maior facilidade de acesso. A desigualdade de distribuição no esforço de coleta impede uma avaliação adequada da distribuição da riqueza florística no Estado, pois o número de espécies reflete mais a densidade de coletas do que a riqueza regional (Shepherd 2003). Além disso, o viés geográfico diminui a acurácia de modelos matemáticos de distribuição geográfica dos taxa, que possuem grande potencial como ferramenta para identificação de áreas prioritárias para conservação (Peterson 2001, Stockwell \& Peterson 2002). Essas limitações reforçam a importância das coletas em número adequado à escala de trabalho e à complexidade do ecossistema em análise.

Estima-se que a flora fanerogâmica paulista compreenda entre 7.500 a 8.000 espécies (Mamede 2003, Shepherd 2003). Das 180 famílias botânicas ocorrentes no estado, 128, (68\%), já receberam tratamento taxonômico até 2007, no contexto do projeto Flora Fanerogâmica do Estado de São Paulo (Wanderley et al. 2001, 2002, 2003, 2005, 2007). Nesse esforço, 61 espécies foram consideradas inéditas e 156 novas ocorrências foram registradas (Wanderley et al. 2001, 2002, 2003, 2005, 2007), evidenciando a elevada riqueza da flora paulista e a necessidade de intensificação das coletas.

A análise dos dados do projeto Flora Fanerogâmica do Estado de São Paulo (FFSP) mostrou uma maior carência de esforço de coleta em municípios situados nas regiões Noroeste, Centro-Oeste e Sudoeste do Estado, esta última correspondendo, em boa parte, à bacia hidrográfica do Alto Paranapanema (Shepherd, dados não publicados). Iniciativas para a avaliação de ações prioritárias para a conservação da biodiversidade da Mata Atlântica e Campos Sulinos, indicam que as ecorregiões abrangidas pelo Alto Paranapanema são consideradas insuficientemente conhecidas, mas de provável importância biológica (MMA 2000). Recentemente, a bacia do Alto Paranapanema foi considerada uma das áreas prioritárias para a execução de levantamentos de flora e fauna no Estado (Rodrigues \& Bononi 2008). Essa região abrange uma área de $20.643 \mathrm{~km}^{2}$ (2.064.300 ha) dos quais 2.979 km² (297.900 ha) são remanescentes de vegetação natural de diversas formações vegetais: Floresta Ombrófila Densa, Ombrófila Mista, Estacional Semidecidual e Cerrado (Kronka et al. 2005). Os levantamentos florísticos realizados na região têm contemplado unidades de conservação como o Parque Estadual de Carlos Botelho (Custódio Filho et al. 1992, Aguiar 2004, Dias 2004) a Estação Ecológica de Angatuba (Torres 1989) e a Estação Experimental de Itararé (Scaramuzza 2006). Contudo, a maior parte das unidades de conservação e municípios da região não foi alvo de estudos sobre a flora. O município de Paranapanema ilustra bem a situação do conhecimento botânico na região. Com uma área de $1.020 \mathrm{~km}^{2}$ (102.000 ha) abriga 7.558 ha de vegetação natural, dos quais 2.183 ha estão protegidos em duas unidades de conservação (Kronka et al. 2005). Não há estudos florísticos detalhados executados nessas unidades ou mesmo no território do município. Uma busca nos acervos dos 
herbários integrantes da rede Species Link (2009) retornou apenas 28 registros correspondentes a coletas realizadas entre 1899 e 1998. A mesma busca, considerando apenas a base SinBiota (Sistema de Informação Ambiental BIOTA/FAPESP), resultou em 295 registros botânicos sem voucher (Species Link 2009).

Este projeto teve como objetivo geral contribuir para a ampliação da densidade de coletas botânicas nessa região, que é uma das menos amostradas do estado de São Paulo. O objetivo específico do projeto foi inventariar e caracterizar a flora vascular nas duas únicas unidades de conservação do município de Paranapanema: Floresta Estadual e Estação Ecológica de Paranapanema. Em conformidade com o objetivo geral, pretende-se futuramente replicar o presente projeto de modo a contemplar outras unidades de conservação na região, consolidando uma linha de pesquisa voltada para o inventário da diversidade vegetal em regiões pouco coletadas do estado de São Paulo.

\section{Material e Métodos}

\section{Localização, clima, relevo, solo e tipo de vegetação}

A Floresta Estadual e a Estação Ecológica de Paranapanema (doravante denominadas área de estudo) são unidades de conservação contíguas situadas no município de mesmo nome, na região Sudoeste do estado de São Paulo (23 32' 02" S e 48 45' 29" O, altitude $630 \mathrm{~m}$ ). Distam aproximadamente $240 \mathrm{~km}$ da capital e $25 \mathrm{~km}$ da sede municipal (Aoki et al. 2001). Situa-se na Depressão Periférica sobre relevo de colinas amplas. O clima regional é Cfa, quente de inverno seco com temperatura média mensal máxima de $22{ }^{\circ} \mathrm{C}$ e média mensal mínima de $18{ }^{\circ} \mathrm{C}$ (Ventura et al. 1965). Entre os tipos de solo, predomina o Latossolo Vermelho Escuro-Orto (Aoki et al. 2001). A área de estudo está localizada na porção central da bacia hidrográfica do Alto Paranapanema e possui 2.183 ha, dos quais 900 ha estão cobertos por Floresta Estacional Semidecidual. A vegetação natural encontra-se segmentada em fragmentos de formato irregular, separados por talhões de Pinus elliottii Engelm. (Figura 1). A Floresta Estacional Semidecidual da área pode ser subdividida em Aluvial e Montana. O detalhamento fitofisionômico, contudo, será alvo de outro estudo que está sendo desenvolvido tendo em vista a elaboração do Plano de Manejo da Estação Ecológica.

\section{2. Área de estudo e coleta de dados}

Todos os fragmentos de vegetação natural foram considerados no inventário florístico, de forma a contemplar os diferentes estádios sucessionais e variações de hábitat da área de estudo. Todas as formas de vida de plantas vasculares foram incluídas no inventário. As trilhas existentes foram georreferenciadas (Tabela 1) com auxílio da Seção de Manejo e Inventário Florestal do Instituto Florestal de São Paulo. Outras trilhas foram abertas e georreferenciadas para permitir a amostragem de toda a cobertura vegetal da área de estudo (Tabela 1). Todas as trilhas foram numeradas e plotadas em um mapa dotado de um sistema de coordenadas geográficas UTM, com as principais vias de acesso e rede de drenagem, de modo a facilitar a sua localização (Figura 1). Para a caracterização florística, a rede de trilhas (totalizando $14,2 \mathrm{~km}$ ) foi percorrida em viagens com duração de uma semana e periodicidade mensal, no período de junho de 2007 a julho de 2008 . O material botânico foi coletado e herborizado conforme Fidalgo \& Bononi (1989). Tanto os materiais férteis como os estéreis foram identificados por meio de comparação com exsicatas depositadas em herbários, consulta à bibliografia e a especialistas (Tabela 2). O material estéril que não passou por especialistas e apresenta taxonomia complexa permaneceu com a identificação cf.

Todo o material fértil coletado foi depositado no Herbário Dom Bento Pickel (SPSF) do Instituto Florestal e os respectivos registros

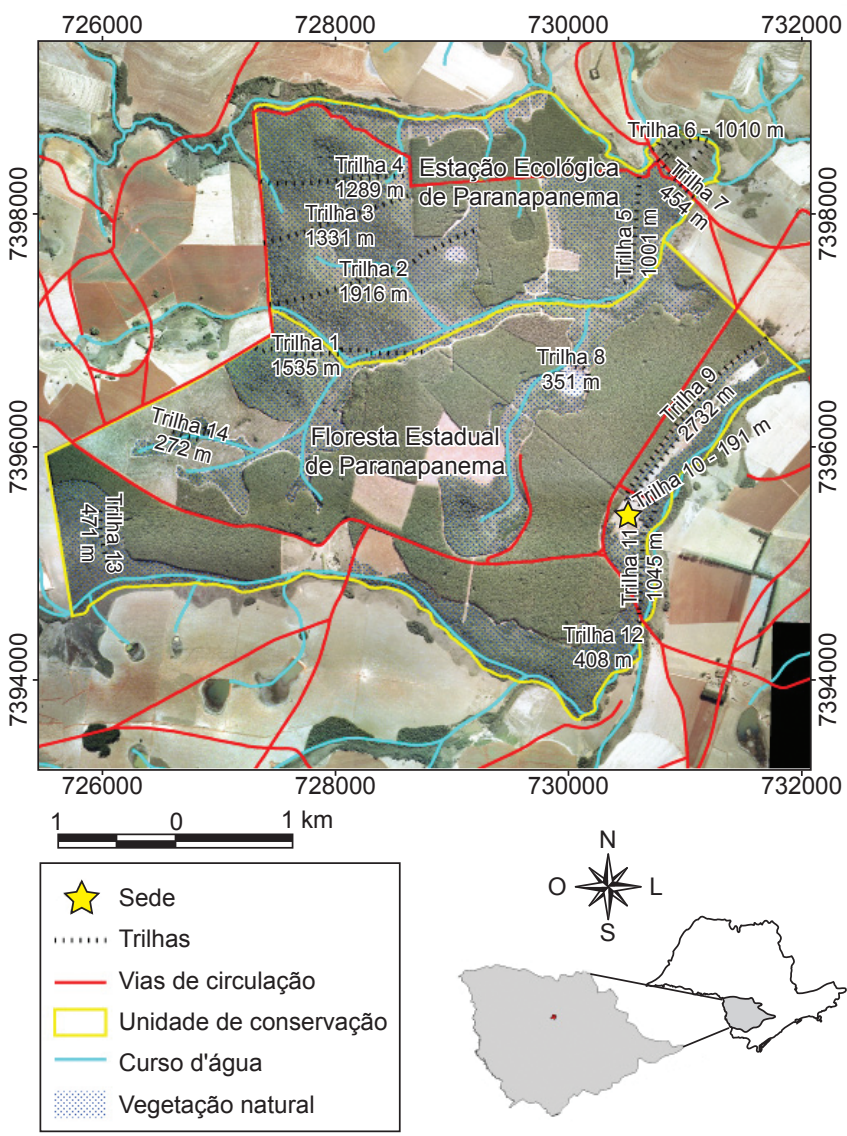

Figura 1. Localização da área de estudo no estado de São Paulo (a área em cinza corresponde à bacia hidrográfica do Alto Paranapanema). Cobertura vegetal natural e trilhas utilizadas na amostragem florística na Floresta Estadual de Paranapanema e Estação Ecológica de Paranapanema. As áreas em verde não hachuradas correspondem a talhões de Pinus elliottii. Fonte: Seção de Manejo e Inventário Florestal, Instituto Florestal de São Paulo.

Figure 1. The study area localization in São Paulo state (the gray area corresponds to the Alto Paranapanema basin). Natural vegetation coverage and the trails used in the floristic sampling at the State Forest of Paranapanema and Ecological Station of Paranapanema. The non-hachured green areas are Pinus elliottii plantations. Source: Section of Management and Forest Inventory, Forest Institute of São Paulo.

estão disponíveis para consulta online (Species Link 2009). O material botânico das espécies coletadas em fase vegetativa foi acondicionado em um acervo próprio e acessível para consultas, nas dependências do mesmo Herbário, constituindo uma coleção testemunha dessas espécies, embora sem número de registro. A lista de espécies que ocorrem na área de estudo foi preparada seguindo o sistema APG II (Judd et al. 2002). A verificação de sinonímias botânicas foi feita por meio de consulta aos banco de dados W3 Tropicos (MOBOT 2008) e International Plant Names Index (IPNI 2008). Foram indicados o número de tombo SPSF, o número da trilha onde a espécie foi coletada e a forma de vida. As coordenadas geográficas dos registros foram consideradas como sendo a das trilhas em que foram coletados (Tabela 1).

Verificou-se o nível de ameaça das espécies através da consulta às listas de espécies ameaçadas de extinção das seguintes fontes:

- Lista Oficial de Espécies Ameaçadas de Extinção no Estado de São Paulo - SMA-SP (São Paulo 2004); 
Tabela 1. Trilhas utilizadas para a amostragem da composição florística na Floresta Estadual de Paranapanema e Estação Ecológica de Paranapanema.

Table 1. Trails used for the floristic composition sampling of the State Forest of Paranapanema and Ecological Station of Paranapanema.

\begin{tabular}{|c|c|c|c|c|}
\hline Trilha & Coordenada (GMS) & Coordenada (UTM) & Ambiente & Extensão (m) \\
\hline 1 & $23^{\circ} 32^{\prime} 21^{\prime \prime} \mathrm{S}$ e $48^{\circ} 46^{\prime} 25^{\prime \prime} \mathrm{O}$ & 727287,7395019 & Floresta Semidecidual & 1535 \\
\hline 2 & $23^{\circ} 31^{\prime} 09^{\prime \prime} \mathrm{S}$ e $48^{\circ} 46^{\prime} 19^{\prime \prime} \mathrm{O}$ & 727492,7397232 & Floresta Semidecidual & 1916 \\
\hline 3 & $23^{\circ} 30^{\prime} 51^{\prime \prime} \mathrm{S}$ e $48^{\circ} 46^{\prime} 21^{\prime \prime} \mathrm{O}$ & 727443,7397786 & Floresta Semidecidual & 1331 \\
\hline 4 & $23^{\circ} 30^{\prime} 35^{\prime \prime} \mathrm{S}$ e $48^{\circ} 46^{\prime} 22^{\prime \prime} \mathrm{O}$ & 727423,7398279 & Floresta Semidecidual & 1289 \\
\hline 5 & $23^{\circ} 30^{\prime} 31^{\prime \prime} \mathrm{S}$ e $48^{\circ} 44^{\prime} 29^{\prime \prime} \mathrm{O}$ & 730631,7398352 & Floresta Semidecidual & 1001 \\
\hline 6 & $23^{\circ} 30^{\prime} 32^{\prime \prime} \mathrm{S}$ e $48^{\circ} 44^{\prime} 18^{\prime \prime} \mathrm{O}$ & 730943,7398316 & Floresta Semidecidual & 1010 \\
\hline 7 & $23^{\circ} 30^{\prime} 28^{\prime \prime} \mathrm{S}$ e $48^{\circ} 44^{\prime} 24^{\prime \prime} \mathrm{O}$ & 730774,7398442 & Floresta Semidecidual & 454 \\
\hline 8 & $23^{\circ} 31^{\prime} 31^{\prime \prime} \mathrm{S}$ e $48^{\circ} 44^{\prime} 44^{\prime \prime} \mathrm{O}$ & 730176,7396513 & Margem de Lagoa & 551 \\
\hline 9 & $23^{\circ} 32^{\prime} 01^{\prime \prime} \mathrm{S}$ e $48^{\circ} 44^{\prime} 31^{\prime \prime} \mathrm{O}$ & 730531,7395584 & Floresta Semidecidual & 2732 \\
\hline 10 & $23^{\circ} 32^{\prime} 03^{\prime \prime} \mathrm{S}$ e $48^{\circ} 44^{\prime} 26^{\prime \prime} \mathrm{O}$ & 730672,7395520 & Floresta Semidecidual & 191 \\
\hline 11 & $23^{\circ} 32^{\prime} 00^{\prime \prime} \mathrm{S}$ e $48^{\circ} 44^{\prime} 23^{\prime \prime} \mathrm{O}$ & 730758,7395611 & Mata Ciliar & 1045 \\
\hline 12 & $23^{\circ} 32^{\prime} 33^{\prime \prime} \mathrm{S}$ e $48^{\circ} 44^{\prime} 28^{\prime \prime} \mathrm{O}$ & 730600,7394598 & Mata Ciliar & 408 \\
\hline 13 & $23^{\circ} 32^{\prime} 23^{\prime \prime} \mathrm{S}$ e $48^{\circ} 44^{\prime} 08^{\prime \prime} \mathrm{O}$ & 725989,7395501 & Floresta Semidecidual & 471 \\
\hline 14 & $23^{\circ} 31^{\prime} 44^{\prime \prime} \mathrm{S}$ e $48^{\circ} 46^{\prime} 49^{\prime \prime} \mathrm{O}$ & 726624,7396168 & Floresta Paludosa & 272 \\
\hline
\end{tabular}

- Revisão da Lista de Espécies da Flora Brasileira Ameaçada de Extinção - FB (Biodiversitas 2008); e

- Lista Vermelha de Espécies Ameaçadas de Extinção da União Internacional para a Conservação da Natureza - IUCN (IUCN 2006).

Optou-se pelo uso da lista da Biodiversitas para a análise das espécies ameaçadas em escala nacional por ser esta lista mais abrangente e ter servido como base para a elaboração da lista oficial de espécies ameaçadas de extinção divulgada recentemente pelo Ministério do Meio Ambiente, através da Instrução Normativa nº 06 de 2008 (Brasil 2008). Contudo, na tabela de espécies ameaçadas da área de estudo as espécies constantes na lista MMA foram sinalizadas.

\section{Resultados}

Foram efetuadas 693 coletas pertencentes a 489 espécies de plantas, sendo que 375 foram identificadas através do exame de material vegetativo e reprodutivo, e 114 espécies foram identificadas com base no exame apenas de material vegetativo. Foram encontrados 294 gêneros e 98 famílias botânicas (Tabela 3). As 489 espécies estão assim distribuídas nas diversas formas de vida: 291 árvores $(59,5 \%)$, 72 lianas (14,7\%), 58 arbustos (11,9\%), 45 ervas (9,2\%), 19 epífitas $(3,9 \%)$ e 4 hemiparasitas $(0,8 \%)$ (Figura 2$)$. Confrontando a lista de espécies do presente estudo com listas de espécies da flora vascular do Cerrado (Mendonça et al. 1998, Castro et al. 1999, Proença et al. 2001) é possível verificar a ocorrência de 138 espécies em comum, das quais 31 espécies são características de Cerrado (Tabela 3).

Considerando-se o total de espécies, independentemente da forma de vida a que pertencem, verificou-se que Myrtaceae (37 espécies), Fabaceae-Faboideae (32), Rubiaceae (28), Bignoniaceae (23), Asteraceae (20), Lauraceae (17), Apocynaceae (15), Melastomataceae (13), Euphorbiaceae, Malvaceae e Fabaceae-Mimosoideae (12 cada), Piperaceae e Rutaceae (10 cada) reuniram 241 espécies ou 49,3\% da riqueza específica da vegetação da área de estudo.

Dentre as arbóreas, Myrtaceae (37 espécies), Fabaceae-Faboideae (26) e Lauraceae (17) foram as mais ricas; Bignoniaceae (15 espécies), Apocynaceae (9) e Asteraceae (7) para lianas; Solanaceae (13), Asteraceae e Piperaceae (6 espécies cada) o foram para as arbustivas; e Cyperaceae (8), Malvaceae (5) e Poaceae (5) para as herbáceas. A maioria das espécies de epífitas concentrou-se na família
Orchidaceae (7 espécies) e, das hemiparasitas, na família Santalaceae (3 espécies).

Dentre as espécies nativas registradas na área de estudo, 15 foram enquadradas em alguma categoria de ameaça (Tabela 4).

\section{Discussão}

Este trabalho resultou em um acréscimo substancial no número de coletas no município de Paranapanema. Considerando as informações disponíveis através da rede Species Link (Species Link 2009), o número de coletas no município de Paranapanema passou de 28 para 721, ou seja, um acréscimo de 693 exsicatas ou 2.475\%. Assim, a densidade de coletas no município passou de 0,027 exsicatas $/ \mathrm{km}^{2}$ para 0,71 exsicatas $/ \mathrm{km}^{2}$. O número de espécies com material testemunho depositado em herbário para o município aumentou de 21 para 388.

A necessidade de ampliação do conhecimento de floras locais e regionais tem sido bastante enfatizada no contexto nacional, em que as lacunas de conhecimento ficam evidentes não só a partir da análise da distribuição do esforço amostral como também de herbários e especialistas (Lewinsohn \& Prado 2002, Peixoto \& Morim 2003, Shepherd 2003, Giulietti et al. 2005, Pirani 2005, Peixoto \& Thomas 2005, Kury 2006). A análise de Shepherd (2003) revela que mesmo em estados das regiões Sul e Sudeste, que concentram o maior número de herbários e especialistas, a densidade de coleta é insatisfatória. Segundo esse autor, no estado de São Paulo, seria necessário dobrar o esforço de coleta de modo a permitir uma avaliação adequada da distribuição da riqueza vegetal (mínimo de 1 exsicata $/ \mathrm{km}^{2}$ ) e sextuplicar esse esforço para atingir um conhecimento completo sobre a flora (mínimo de 3 exsicatas $/ \mathrm{km}^{2}$ ). A densidade obtida com este trabalho $\left(0,71\right.$ exsicatas $\left./ \mathrm{km}^{2}\right)$, portanto, está abaixo dos valores preconizados. Contudo, a densidade de coletas no nível de município não é o único critério para planejar a alocação do esforço de coleta. No caso do município de Paranapanema, um aumento na densidade de coletas seria desejável, em função da ocorrência de áreas de Cerrado no município, fitofisionomia não contemplada neste trabalho. Porém, levantamentos florísticos em Cerrado poderiam ser feitos em municípios vizinhos, permitindo igualmente a elevação do conhecimento sobre a flora regional e resultando em maior uniformidade na distribuição espacial do esforço de coleta. Portanto, a distribuição espacial do esforço de 
Tabela 2. Lista de especialistas e respectivas famílias, consultados na fase de identificação dos exemplares botânicos coletados na Floresta Estadual de Paranapanema e Estação Ecológica de Paranapanema.

Table 2. List of taxonomists, and respective families, consulted during the identification phase of the botanical specimens collected at the State Forest of Paranapanema and Ecological Station of Paranapanema.

\begin{tabular}{|c|c|c|}
\hline Família & Especialista & Instituição \\
\hline Acanthaceae & Cíntia Kameyama & Instituto de Botânica de São Paulo \\
\hline Asteraceae & João Semir & Universidade Estadual de Campinas \\
\hline Asteraceae & Marcelo Monge Egea & Universidade Estadual de Campinas \\
\hline Bromeliaceae & Anderson Santos & Instituto de Botânica de São Paulo \\
\hline Bromeliaceae & Luciana Fiorato & Instituto de Botânica de São Paulo \\
\hline Cactaceae & Eduardo Luis Catharino & Instituto de Botânica de São Paulo \\
\hline Commelinaceae & Lidyanne Yuriko Saleme Aona & Univ. Federal do Recônvavo Baiano \\
\hline Convolvulaceae & Rosângela Simão Bianchini & Instituto de Botânica de São Paulo \\
\hline Cyatheaceae & Jefferson Prado & Instituto de Botânica de São Paulo \\
\hline Cyperaceae & André Gil & Universidade Estadual de Campinas \\
\hline Euphorbiaceae & Inês Cordeiro & Instituto de Botânica de São Paulo \\
\hline Fabaceae-Caesalpinioideae & Marcos José da Silva & Universidade Estadual de Campinas \\
\hline Fabaceae-Cercideae & Ana Maria G. de Azevedo Tozzi & Universidade Estadual de Campinas \\
\hline Fabaceae-Faboideae & Vidal Mansano & Jardim Botânico do Rio de Janeiro \\
\hline Fabaceae-Faboideae & Marcos José da Silva & Universidade Estadual de Campinas \\
\hline Fabaceae-Mimosoideae & Marcos José da Silva & Universidade Estadual de Campinas \\
\hline Lauraceae & João Batista Baitello & Instituto Florestal de São Paulo \\
\hline Malpighiaceae & Maria Cândida Henrique Mamede & Instituto de Botânica de São Paulo \\
\hline Malpighiaceae & Renata Sebastiani & Instituto de Botânica de São Paulo \\
\hline Malvaceae & Gerlaine Esteves & Instituto de Botânica de São Paulo \\
\hline Melastomataceae & Renato Goldenberg & Universidade Federal do Paraná \\
\hline Melastomataceae & Ângela Borges Martins & Universidade Estadual de Campinas \\
\hline Meliaceae & João Aurélio Pastore & Instituto Florestal de São Paulo \\
\hline Moraceae & Sergio Romaniuc Neto & Instituto de Botânica de São Paulo \\
\hline Moraceae & André Gaglioti & Instituto de Botânica de São Paulo \\
\hline Myrsinaceae & Luis Carlos Bernacci & Instituto Agronômico de Campinas \\
\hline Myrtaceae & Osny Tadeu de Aguiar & Instituto Florestal de São Paulo \\
\hline Ochnaceae & Kikyo Yamamoto & Universidade Estadual de Campinas \\
\hline Orchidaceae & Fábio de Barros & Instituto de Botânica de São Paulo \\
\hline Passifloraceae & Luis Carlos Bernacci & Instituto Agronômico de Campinas \\
\hline Phyllanthaceae & Inês Cordeiro & Instituto de Botânica de São Paulo \\
\hline Piperaceae & Else Guimarães & Jardim Botânico do Rio de Janeiro \\
\hline Poaceae & Carlos Garcia Santos & Universidade de São Paulo \\
\hline Poaceae & Paulo Takeo Sano & Universidade de São Paulo \\
\hline Polypodiaceae & Jefferson Prado & Instituto de Botânica de São Paulo \\
\hline Pteridaceae & Jefferson Prado & Instituto de Botânica de São Paulo \\
\hline Rubiaceae & Sigrid Jung-Mendaçolli & Instituto Agronômico de Campinas \\
\hline Rutaceae & José Rubens Pirani & Universidade de São Paulo \\
\hline Salicaceae & Roseli Torres & Instituto Agronômico de Campinas \\
\hline Schizaeaceae & Jefferson Prado & Instituto de Botânica de São Paulo \\
\hline Solanaceae & João Renato Stehmann & Universidade Federal de Minas Gerais \\
\hline Styracaceae & Reinaldo Monteiro & Universidade Estadual Paulista \\
\hline Symplocaceae & João Aranha & Universidade Estadual de Campinas \\
\hline Thymelaeaceae & Lucia Rossi & Instituto de Botânica de São Paulo \\
\hline Vochysiaceae & Kikyo Yamamoto & Universidade Estadual de Campinas \\
\hline Vochysiaceae & Gustavo Shimizu & Universidade Estadual de Campinas \\
\hline
\end{tabular}




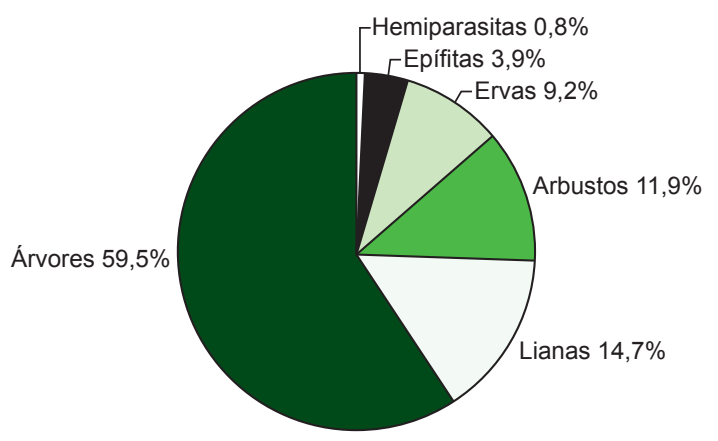

Figura 2. Distribuição de frequiência de formas de vida das espécies encontradas na Floresta Estadual de Paranapanema e Estação Ecológica de Paranapanema.

Figure 2. Frequency distribution of life forms of the species found at the State Forest of Paranapanema and Ecological Station of Paranapanema.

coleta também deveria ser considerada. Assim, o presente projeto está tendo continuidade atualmente na Estação Ecológica de Itapeva, no município de Itapeva, em área de Cerrado.

O estado de São Paulo possui 645 municípios com área média de $386 \mathrm{~km}^{2}$ (Coeficiente de Variação $=83 \%$ ). Devido ao tamanho relativamente pequeno dos municípios, a tendência é haver maior homogeneidade na distribuição espacial do esforço amostral quando o planejamento das coletas botânicas é feito ao nível de município. Uma busca simples na rede Species Link possibilita a obtenção de informações sobre o número de registros de coletas botânicas nos municípios da região da bacia hidrográfica e o estabelecimento de um rol de municípios prioritários para a alocação do esforço de coleta. No estabelecimento desse rol todos os tipos vegetacionais da região devem ser contemplados, bem como devem ser ponderadas a ocorrência de remanescentes naturais nos municípios e a homogeneidade na distribuição espacial do esforço de coleta. Estes critérios também precisam ser compatibilizados com aspectos de ordem prática como a facilidade de acesso, a disponibilidade de infra-estrutura de pesquisa e a presença de unidades de conservação. Infelizmente, boa parte dos herbários do estado de São Paulo não disponibiliza integralmente suas coleções através da base Species Link. Assim, a avaliação da densidade de coletas botânicas no nível de município utilizando aquela base de dados deve ser feita com cautela, procurando-se verificar a ocorrência de exsicatas provenientes de coletas realizadas nos municípios avaliados no acervo de herbários da região.

Considerando-se todas as formas de vida, o número de espécies (489) e famílias (98) verificado na amostra de composição florística da área de estudo foi superior ao obtido em inventários semelhantes realizados em outras florestas semideciduais no estado de São Paulo, que apresentaram entre 148 e 362 espécies e 46 e 95 famílias (Bernacci \& Leitão Filho 1996, Stranghetti \& Ranga 1998, Kinoshita et al. 2006). Mesmo considerando-se apenas os componentes arbóreo e arbustivo, que totalizaram 349 espécies, verifica-se que o número de espécies obtido para a área de estudo é superior ao relatado em outros levantamentos voltados para essas formas de vida, e que obtiveram entre 61 e 175 espécies arbustivo-arbóreas (Ivanauskas et al. 1999, Fonseca \& Rodrigues 2000, Durigan et al. 2000, Cielo-Filho \& Santin 2002, Santos \& Kinoshita 2003, Silva \& Soares 2003, Yamamoto et al. 2005). Esses levantamentos foram realizados em diversas localidades do estado de São Paulo e a porção de vegetação natural das áreas de estudo variou entre 2 ha e 2.000 ha.

Além das diferenças no tamanho das áreas amostradas, os prováveis motivos para o número relativamente elevado de espécies desta área de estudo podem ser o bom estado de conservação da vegetação, a variedade de hábitat e sua localização em uma região de interface entre várias formações vegetais que ocorrem no estado de São Paulo (Kronka et al. 2005). Embora não tenha sido detectada a fitofisionomia Cerrado na área de estudo, várias espécies características de Cerrado foram encontradas, especialmente sob plantios de Pinus elliottii. Em estudo realizado no município de Itatinga, SP, Ivanauskas et al. (1999) mencionam possíveis particularidades da flora regional, devido à sua localização em áreas de interface entre várias formações vegetais e ao inverno mais rigoroso.

Quanto à distribuição da riqueza nas diversas formas de vida, predominaram plantas de hábito arbóreo, que são cerca de $60 \%$ do total das espécies. Lianas, arbustos, ervas, epífitas e hemiparasitas reuniram de 0,8 a $14,7 \%$ das espécies, perfazendo os $40 \%$ que correspondem à flora não-arbórea da área de estudo. Outros levantamentos amplos em florestas estacionais semideciduais indicaram que as árvores foram a forma de vida com maior número de espécies (entre 33 e $37 \%$ do total) e que as proporções com que ocorreram cada uma das diversas formas de vida não-arbóreas como lianas, arbustos e ervas variaram entre 5,2 e 39\% (Bernacci \& Leitão Filho 1996, Stranghetti \& Ranga 1998; Kinoshita et al. 2006). Diferenças nos esforços e épocas de coleta, objetivos dos estudos e estado de conservação da vegetação podem influenciar os resultados de levantamentos deste tipo, dificultando comparações muito precisas entre áreas. Além disso, a falta de um maior número de inventários amplos dificulta o reconhecimento de um padrão de distribuição de riqueza de espécies nas diferentes formas de vida. Considerando os dados deste trabalho e os disponíveis na literatura, contudo, pode-se afirmar que as formas de vida não-arbóreas contribuem com algo em torno de $43 \%$ da riqueza de espécies na Floresta Estacional Semidecídua paulista. Ivanauskas et al. (2001) e Ziparro et al. (2005) encontraram contribuições de espécies não-arbóreas para a composição florística de florestas ombrófilas paulistas entre 43 e $51 \%$, o que sugere que as florestas ombrófilas e estacionais do Estado são semelhantes no que se refere à contribuição do componente não-arbóreo para a riqueza florística total.

Em relação à distribuição de espécies nas famílias botânicas, os resultados deste estudo corroboraram as informações disponíveis na literatura. Para o componente arbustivo, a ocorrência expressiva de Fabaceae, Myrtaceae, Euphorbiaceae, Rutaceae, Meliaceae, Lauraceae e Rubiaceae foi relatada em publicações recentes para diversas regiões do estado (Ivanauskas et al. 1999, Fonseca \& Rodrigues 2000, Durigan et al. 2000, Cielo-Filho \& Santin 2002, Santos \& Kinoshita 2003, Silva \& Soares 2003, Yamamoto et al. 2005, Kinoshita et al. 2006). Além de ampliarem o conhecimento sobre a flora das florestas semideciduais paulistas e fornecerem informações sobre a distribuição da diversidade vegetal remanescente, esses levantamentos têm permitido confirmar o padrão de riqueza de famílias nas florestas estacionais, corroborando as informações de Leitão Filho $(1982,1987)$ e de outros trabalhos clássicos realizados nas décadas de 1980 e 1990, conforme comparações realizadas por Santos \& Kinoshita (2003) e Kinoshita et al. (2006). É consenso nesses trabalhos a constatação de que poucas famílias reúnem a maioria das espécies presentes nas áreas estudadas, como também verificado na área de estudo, onde cerca de 50\% das espécies pertencem a apenas 13 famílias.

Levantamentos que consideraram as lianas relataram a ocorrência de 35 a 148 espécies, demonstrando a alta concentração de espécies em poucas famílias, como Bignoniaceae, Sapindaceae, Malpighiaceae, Apocynaceae, Fabaceae e Convolvulaceae, que caracterizam a comunidade desta forma de vida e são as mais ricas em espécies na maioria das florestas semidecíduas estudadas no estado de São Paulo (Bernacci \& Leitão Filho 1996, Stranghetti \& Ranga 
Tabela 3. Espécies de plantas vasculares da Floresta Estadual de Paranapanema e Estação Ecológica de Paranapanema. Formas de vida: Av, árvore; Ab, arbusto; Ev, erva; Li, liana; Ep, epífita; He, hemiparasita. Espécies características de Cerrado estão indicadas por um asterisco. SPSF, número de tombo no Herbário Dom Bento Pickel.

Table 3. Vascular plant species of the State Forest of Paranapanema and Ecological Station of Paranapanema. Life forms: Av, tree; Ab, shrub; Ev, herb; Li, liana; He, hemiparasite. Species characteristics of the Cerrado (Brazilian savanna) are indicated with an asterisk. SPSF, specimen record number at the Dom Bento Pickel Herbarium.

\begin{tabular}{|c|c|c|c|c|}
\hline Família/Espécie & Nome popular & FV & Trilha & SPSF \\
\hline \multicolumn{5}{|l|}{ ACANTHACEAE } \\
\hline Geissomeria schottiana Nees & & $\mathrm{Li}$ & $1,4,5$ & $38091,38293,38657$ \\
\hline Ruellia angustiflora (Nees) Lind. ex Rambo & alfavaca-de-cobra & $\mathrm{Ab}$ & 1,2 & 38191,38535 \\
\hline \multicolumn{5}{|l|}{ AMARANTHACEAE } \\
\hline Hebanthe paniculata Mart. & corango-açu & $\mathrm{Ab}$ & 2,13 & 38566,38709 \\
\hline \multicolumn{5}{|l|}{ ANACARDIACEAE } \\
\hline Astronium graveolens Jacq. & guaritá & Av & 2,1 & \\
\hline Lithraea molleioides (Vell.) Engl. & aroeira-brava & $\mathrm{Av}$ & $1,3,4$ & $38192,38114,38677,38881$ \\
\hline Schinus terebinthifolius Raddi* & aroeira-pimenteira & $\mathrm{Av}$ & $1,6,14$ & $38935,39220,39227$ \\
\hline Tapirira guianensis Aublet. & peito-de-pombo & $\mathrm{Av}$ & $1,4,5$ & 38886,38888 \\
\hline \multicolumn{5}{|l|}{ ANNONACEAE } \\
\hline Annona cacans Warm. & araticum-cagão & Av & 11 & \\
\hline Annona cf. crotonifolia Mart. & araticum & Av & 1 & \\
\hline Duguetia furfuracea (A.St.-Hil.) Benth. \& Hook. f.* & marolinho & $\mathrm{Av}$ & & 38526,38980 \\
\hline Duguetia lanceolata A.St.-Hil. & pindaíba & Av & $1,2,3,4,5$ & 39249 \\
\hline Guatteria nigrescens Mart. & pindaíba-preta & Av & 3,9 & 38799 \\
\hline Porcelia macrocarpa (Warm.) R.E.Fries & louro-branco & $\mathrm{Av}$ & 1 & \\
\hline Rollinia emarginata Schltdl. & pindaíba & Av & $1,9,12$ & 39798 \\
\hline Rollinia sericea R.E.Fries & araticum-alvadio & $\mathrm{Av}$ & 1 & \\
\hline Xylopia brasiliensis (L.) Spreng.* & pindaíba & Av & 4 & \\
\hline \multicolumn{5}{|l|}{ APOCYNACEAE } \\
\hline Asclepias curassavica $\mathrm{L}$. & oficial-de-sala & $\mathrm{Ab}$ & & 38513 \\
\hline Aspidosperma australe Müll.Arg. & pequiá & Av & 6 & 38073 \\
\hline Aspidosperma camporum Müll.Arg. & pequiá & $\mathrm{Av}$ & 5,3 & \\
\hline Aspidosperma cylindrocarpon Mart. & peroba-poca & $\mathrm{Av}$ & $3,4,12$ & 38675,38753 \\
\hline Aspidosperma polyneuron Müll.Arg. & peroba-rosa & $\mathrm{Av}$ & 1 & \\
\hline Blepharodon bicuspidatum E.Fourn. & & $\mathrm{Li}$ & 5 & 39252 \\
\hline Condylocarpon isthmicum (Vell.) A.DC. & cipó-de-leite & $\mathrm{Li}$ & $4,6,14$ & 38132,38940 \\
\hline Forsteronia glabrescens Müll. Arg. & & $\mathrm{Li}$ & 14 & 39219 \\
\hline Forsteronia velloziana (A.DC.) Woodson & & $\mathrm{Li}$ & 4 & 38115 \\
\hline Gonioanthela axillaris (Vell.) Fontella \& E.A.Schwarz & & $\mathrm{Li}$ & 2,4 & 38081,38196 \\
\hline Mesechites mansoanus (A.DC.) Woodson & & $\mathrm{Li}$ & 5 & 39201 \\
\hline Orthosia urceolata E.Fourn. & & $\mathrm{Li}$ & 6 & 38938 \\
\hline Prestonia caalita (Vell.) Woodson & cipó-de-leite & $\mathrm{Li}$ & 8 & 39198 \\
\hline Prestonia riedelii (Müll.Arg.) Markgr. & cipó-capoeira & $\mathrm{Li}$ & 3 & 38523,38650 \\
\hline Tabernaemontana laeta Mart. & café-do-mato & Av & $2,10,7,9$ & $38932,38118,38910$ \\
\hline \multicolumn{5}{|l|}{ AQUIFOLIACEAE } \\
\hline Ilex amara (Vell.) Loes. & caúna-lisa & Av & 1,4 & \\
\hline Ilex brasiliensis (Spreng.) Loes. & mate-falso & Av & 4 & \\
\hline Ilex brevicuspis Reiss. & caúna-da-serra & $\mathrm{Av}$ & 1 & \\
\hline Ilex paraguariensis A.St.-Hil. & erva-mate & $\mathrm{Av}$ & $2,5,8$ & 38195,38200 \\
\hline \multicolumn{5}{|l|}{ ARALIACEAE } \\
\hline Dendropanax cuneatus (DC.) Decne. \& Planch. & maria-mole & $\mathrm{Av}$ & $1,3,4$ & $\begin{array}{l}38190,38199,38109,38521, \\
38548,38674,38615\end{array}$ \\
\hline $\begin{array}{l}\text { Schefflera cf. morototoni (Aubl.) } \\
\text { Maguire, Steyermark \& Frodin }\end{array}$ & morototó & Av & 2,3 & \\
\hline
\end{tabular}


Cielo-Filho, R. et al.

Tabela 3. Continuação...

\begin{tabular}{|c|c|c|c|c|}
\hline Família/Espécie & Nome popular & FV & Trilha & SPSF \\
\hline \multicolumn{5}{|l|}{ ARECACEAE } \\
\hline Astrocaryum aculeatissimum (Schott) Burret & brejaúva & Av & 3 & \\
\hline Butia archeri (Glassman) Glassman* & butiá & Av & & 38589 \\
\hline Euterpe edulis Mart. & palmito & Av & 14 & \\
\hline Geonoma schottiana Mart. & gamiova & $\mathrm{Av}$ & $7,12,14$ & $38564,38974,39205$ \\
\hline Syagrus romanzoffiana (Cham.) Glassman & jerivá & $\mathrm{Av}$ & $1,2,3,4,5$ & \\
\hline \multicolumn{5}{|l|}{ ARISTOLOCHIACEAE } \\
\hline Aristolochia esperanzae Kuntze & jarrinha-do-cerrado & $\mathrm{Li}$ & 14 & 39228 \\
\hline Aristolochia sp. & papo-de-peru & $\mathrm{Li}$ & 1 & 38653 \\
\hline \multicolumn{5}{|l|}{ ASTERACEAE } \\
\hline Ageratum conyzoides L.* & mentrasto & Ev & 3 & 38129 \\
\hline Baccharis brachylaenoides DC. & vassoura & $\mathrm{Ab}$ & 2,5 & 38076,38197 \\
\hline Baccharis cf. semiserrata Baker & vassoura & $\mathrm{Ab}$ & 3 & \\
\hline Baccharis trimera (Less.) DC. & carqueja & $\mathrm{Ab}$ & 7 & 38593 \\
\hline Baccharis trinervis Pers. & assapeixe-fino & $\mathrm{Ab}$ & 2,7 & 38587,38568 \\
\hline Calea pinnatifida (R.Br.) Baker & erva-de-lagarto & $\mathrm{Li}$ & 3,7 & 38203,38720 \\
\hline Dasyphyllum brasiliense (Spreng.) Cabrera & guaiapá-parreira & $\mathrm{Ab}$ & $1,4,7,9$ & 38530,38572 \\
\hline Gochnatia polymorpha (Less.) Cabrera* & cambará & $\mathrm{Av}$ & $2,3,4,7$ & $\begin{array}{l}38082,38204,38201,38931, \\
38920\end{array}$ \\
\hline Heterocondylus vitalbae (DC.) R.M.King \& H.Robins & & $\mathrm{Li}$ & 1 & 38632 \\
\hline Mikania glomerata Spreng. & guaco-verdadeiro & $\mathrm{Li}$ & 1 & 38194 \\
\hline Mikania hirsutissima DC. & guaco-cabeludo & $\mathrm{Li}$ & 7 & 38597 \\
\hline Mikania micrantha Kunth & guaco & $\mathrm{Li}$ & 4 & 38123 \\
\hline Mikania miyriocephala DC. & cundurango & $\mathrm{Li}$ & 4 & 38086 \\
\hline Mutisia coccinea A.St.-Hil. & cravo-divino-branco & $\mathrm{Li}$ & 2 & 38538 \\
\hline Piptocarpha angustifolia Dusén ex Malme & vassourão-branco & Av & 7 & 38812 \\
\hline Piptocarpha axillaris (Less.) Baker & vassourão & Av & $1,5,7$ & $38111,38510,38761,38643$ \\
\hline Piptocarpha sellowii (Sch. Bip.) Baker & & Av & 5 & \\
\hline Sphagneticola trilobata (L.) Pruski & margaridão & Ev & & 38793 \\
\hline Symphyopappus cuneatus (DC.) Sch.Bip. ex Baker & eupatório & $\mathrm{Ab}$ & 5 & 38103 \\
\hline Vernonia muricata DC. & & Ev & 4 & 38139 \\
\hline \multicolumn{5}{|l|}{ BIGNONIACEAE } \\
\hline Adenocalymma bracteatum (Cham.) DC. & cipó-branco & $\mathrm{Li}$ & 2,9 & 38598,38528 \\
\hline Arrabidaea blanchetii DC. & & $\mathrm{Li}$ & 2 & 38122 \\
\hline Arrabidaea chica (Humb. \& Bonpl.) B. Verl & carajurú & $\mathrm{Li}$ & 7 & $38802,38810,38739,38727$ \\
\hline Arrabidaea craterophora (DC.) Bureau & arrabidea-rosa & $\mathrm{Li}$ & 9 & 38594 \\
\hline Arrabidaea leocopogon Cham. & cipó-camarão-branco & $\mathrm{Li}$ & 6,9 & 38999,38993 \\
\hline Arrabidaea pubescens (L.) A.H. Gentry & & $\mathrm{Li}$ & 14 & 39218 \\
\hline Arrabidaea selloi (Spreng.) Sandwith & cipó-camarão-de-selo & $\mathrm{Li}$ & 1,6 & 38207,38957 \\
\hline Arrabidaea triplinervia Mart. ex DC. & & $\mathrm{Li}$ & $4,5,10$ & 38128,38911 \\
\hline Arrabidaea sp. & cipó-cruz & $\mathrm{Li}$ & 1 & 38893 \\
\hline Cuspidaria floribunda (DC.) A.H. Gentry & cuspidária & $\mathrm{Li}$ & 13 & 38787 \\
\hline Cuspidaria pterocarpa (Cham.) DC. & cuspidária & $\mathrm{Li}$ & 6 & 38954 \\
\hline Cybistax antisyphilitica (Mart.) Mart.* & ipê-verde & Av & 3 & \\
\hline Fridericia speciosa Mart. & cipó-vermelho & $\mathrm{Li}$ & 2,9 & $38209,38976,39255,38900$ \\
\hline Handroanthus chrysotrichus (Mart. ex A. DC.) Mattos & ipê-amarelo & $\mathrm{Av}$ & 2 & \\
\hline Handroanthus heptaphyllus (Vell.) Mattos & ipê-roxo & $\mathrm{Av}$ & 5 & \\
\hline Handroanthus ochraceus (Cham.) Mattos* & ipê-amarelo & Av & $1,2,5$ & 38646 \\
\hline Jacaranda micrantha Charm. & caroba-da-mata & Av & 10 & 38909 \\
\hline Jacaranda oxyphylla Cham. & caroba-de-são-paulo & $\mathrm{Ab}$ & 4 & 38654 \\
\hline
\end{tabular}


Tabela 3. Continuação...

\begin{tabular}{|c|c|c|c|c|}
\hline Família/Espécie & Nome popular & FV & Trilha & SPSF \\
\hline Jacaranda puberula Cham.* & carobinha & Av & 1 & \\
\hline Macfadyena unguis-cati L. & unha-de-gato & $\mathrm{Li}$ & 10 & 38992 \\
\hline Pithecoctenium sp. & pente-de-macaco & $\mathrm{Li}$ & 1 & 38283 \\
\hline Pyrostegia venusta (Ker Gawl.) Miers. & cipó-de-são-joão & $\mathrm{Li}$ & 2 & 38543 \\
\hline Zeyheria tuberculosa Burr. & ipê-tabaco & Av & 4 & \\
\hline \multicolumn{5}{|l|}{ BORAGINACEAE } \\
\hline Cordia eucalyculata Vell. & café-de-bugre & $\mathrm{Av}$ & 11 & \\
\hline Cordia sellowiana Cham. & chá-de-bugre & $\mathrm{Av}$ & $3,6,7,9$ & $\begin{array}{l}38075,38527,38533,38678, \\
38716,38969\end{array}$ \\
\hline Cordia superba Cham. & babosa-branca & $\mathrm{Av}$ & $6,7,11,12$ & 38968,38945 \\
\hline Cordia trichotoma (Vell.) Arrab. ex Steud. & louro-pardo & $\mathrm{Av}$ & 3 & 38112 \\
\hline Patagonula americana $\mathrm{L}$. & guaiuvira & Av & 1 & \\
\hline Tournefortia paniculata Cham. & marmelinho & $\mathrm{Li}$ & 9 & 38941 \\
\hline \multicolumn{5}{|l|}{ BROMELIACEAE } \\
\hline Aechmea distichantha Lem.* & caraguatá & Ep & 13 & 38786 \\
\hline Bilbergia distachia (Vell.) Mez & gravatá & Ep & 2,3 & 38537,38676 \\
\hline Bromelia balansae $\mathrm{Mez}^{*}$ & caraguatá & Ev & 3 & \\
\hline Tillandsia tenuifolia $\mathrm{L}$. & tilandsia & Ep & 2 & 38208,38212 \\
\hline \multicolumn{5}{|l|}{ BURSERACEAE } \\
\hline Protium heptaphyllum Mart. & almecega & Av & 7,5 & \\
\hline Protium cf. spruceanum (Benth.) Engl. & almecega-do-brejo & $\mathrm{Av}$ & 1 & \\
\hline \multicolumn{5}{|l|}{ CACTACEAE } \\
\hline Cereus hildmanianus K. Schum. & mandacaru & Av & 3 & \\
\hline Lepismium cruciforme (Vell.) Miq. & cruzeta & Ep & 4 & 38621 \\
\hline Rhipsalis cereuscula Haw. & comambaia & Ep & 1 & 38206,38896 \\
\hline Rhipsalis floccosa Salm-Dyck ex Pfeiffer & conambaia & Ep & 4 & 38660 \\
\hline \multicolumn{5}{|l|}{ CAMPANULACEAE } \\
\hline Lobelia camporum Pohl* & voadeira-do-brejo & Ev & 9 & 38979 \\
\hline \multicolumn{5}{|l|}{ CANNABACEAE } \\
\hline Celtis iguanaea (Jacq.) Sarg. & jameri & $\mathrm{Li}$ & 2,3 & 38534,38667 \\
\hline Celtis spinosa Spreng. & esporão & $\mathrm{Ab}$ & 1 & \\
\hline Trema micrantha Cham & crindeúva & $\mathrm{Av}$ & 9 & 38981 \\
\hline \multicolumn{5}{|l|}{ CANNACEAE } \\
\hline Canna coccinea Mill. & cana-dos-jardins & Ev & 1 & 38205 \\
\hline \multicolumn{5}{|l|}{ CARDIOPTERIDACEAE } \\
\hline Citronella paniculata (Mart.) R.A.Howard & falsa-congonheira & $\mathrm{Av}$ & $11,13,12$ & \\
\hline \multicolumn{5}{|l|}{ CELASTRACEAE } \\
\hline Hippocratea volubilis L. & cipó-preto & $\mathrm{Li}$ & 9 & 38998 \\
\hline Maytenus aquifolium Mart. & espinheira-santa & Av & 5,1 & \\
\hline Maytenus evonymoides Reissek & laranjinha & $\mathrm{Av}$ & $1,3.7$ & $38725,38751,38929$ \\
\hline Maytenus robusta Reissek & cafezinho & $\mathrm{Av}$ & 1,2 & 38211 \\
\hline Peritassa campestris (Camb.) A.C.Smith* & bacupari & $\mathrm{Ab}$ & & \\
\hline \multicolumn{5}{|l|}{ CHRYSOBALANACEAE } \\
\hline Hirtella hebeclada Moric. & macucurana & $\mathrm{Av}$ & 2 & \\
\hline \multicolumn{5}{|l|}{ CLETHRACEAE } \\
\hline Clethra scabra Pers. & carne-de-vaca & $\mathrm{Av}$ & $1,2,4$ & 38074 \\
\hline \multicolumn{5}{|l|}{ CHLORANTHACEAE } \\
\hline Hedyosmum brasiliense Miq. & chá-de-soldado & $\mathrm{Av}$ & 14 & 39207 \\
\hline \multicolumn{5}{|l|}{ COMBRETACEAE } \\
\hline Terminalia triflora (Griseb.) Lillo & amarelinho & Av & $3,6,12,14$ & 39009 \\
\hline
\end{tabular}


Cielo-Filho, R. et al.

Tabela 3. Continuação...

\begin{tabular}{|c|c|c|c|c|}
\hline Família/Espécie & Nome popular & FV & Trilha & SPSF \\
\hline \multicolumn{5}{|l|}{ COMMELINACEAE } \\
\hline Dichorisandra hexandra (Aubl.) Kuntze ex Hand.-Mazz. & & Ev & 8 & 39260 \\
\hline \multicolumn{5}{|l|}{ CONVOLVULACEAE } \\
\hline Ipomoea aristolochiifolia $\mathrm{G}$. Don & campainha & Ev & 3 & 38098 \\
\hline Jacquemontia blanchetii Moric. & campainha & $\mathrm{Li}$ & 7 & 38921 \\
\hline \multicolumn{5}{|l|}{ CUNONIACEAE } \\
\hline Lamanonia ternata Vell. & cangalheiro & Av & 2 & 38214 \\
\hline \multicolumn{5}{|l|}{ CYATHEACEAE } \\
\hline Alsophila sternbergii (Sternb.) Conant. & samambaiaçu & Av & 1 & 38644 \\
\hline Cyathea atrovirens (Langsd. \& Fisch.) Domin & samambaiaçu & Av & 1 & 38447 \\
\hline \multicolumn{5}{|l|}{ CYPERACEAE } \\
\hline Cyperus haspan $\mathrm{L}$. & tiririca & Ev & 7 & 38508 \\
\hline Eleocharis nudipes (Kunth) Palla & & Ev & 8 & 38600 \\
\hline Rhynchospora corymbosa (L.) Britton & capituva & Ev & 8 & 38602 \\
\hline Rhynchospora exaltata Kunth & periperi & Ev & 2,14 & 38213,39202 \\
\hline Rhynchospora legrandii Kük. & & Ev & 2 & 38560 \\
\hline Rhynchospora splendens Lindman & & Ev & 7 & 38596 \\
\hline Scleria latifolia $\mathrm{Sw}$. & & Ev & 14 & 39204 \\
\hline Scleria plusiophylla Steud. & & Ev & 3 & 38210 \\
\hline \multicolumn{5}{|l|}{ DILLENIACEAE } \\
\hline Davilla rugosa Poir. & cipó-cabloco & $\mathrm{Li}$ & $1,2,4,7$ & 38138,38544 \\
\hline \multicolumn{5}{|l|}{ EBENACEAE } \\
\hline Diospyros inconstans Jacq. & marmelinho & Av & 1,7 & 38982 \\
\hline \multicolumn{5}{|l|}{ ELAEOCARPACEAE } \\
\hline Sloanea monosperma Vell. & ouriço & Av & $3,2,5$ & \\
\hline \multicolumn{5}{|l|}{ ERICACEAE } \\
\hline Gaylussacia brasiliensis (Spreng.) Meisn. var. brasiliensis & camarinha & $\mathrm{Ab}$ & 14 & 39231 \\
\hline \multicolumn{5}{|c|}{ ERYTHROXYLACEAE } \\
\hline Erythroxylum campestre A.St.-Hil.* & mercúrio & $\mathrm{Ab}$ & & 38916 \\
\hline Erythroxylum cuneifolium (Mart.) O.E.Schulz* & mercúrio & Av & $13,6,7$ & $38811,38740,38946,38928$ \\
\hline Erythroxylum deciduum A.St.-Hil.* & cocão & Av & 2 & 38732 \\
\hline \multicolumn{5}{|l|}{ EUPHORBIACEAE } \\
\hline Acalypha gracilis Müll.Arg. & acalifa & $\mathrm{Ab}$ & 7 & 38958 \\
\hline Actinostemon concolor (Spreng.) Müll.Arg. & limoeiro-do-mato & Av & $1,4,12$ & 38219,38663 \\
\hline Actinostemon klotzschii (Didr.) Pax & limão-bravo & Av & $1,13,7$ & $38218,38515,38634,38744$ \\
\hline Bernardia pulchella (Baill.) Müll.Arg. & & $\mathrm{Ab}$ & 4,9 & $38221,38225,38898$ \\
\hline Croton floribundus Spreng. & capixingui & Av & 9,8 & 38899,39199 \\
\hline Croton urucurana Baill. & sangra-d'água & Av & 10 & 38908 \\
\hline Sapium glandulatum (Vell.) Pax & leiteiro & Av & 10 & \\
\hline Sebastiania brasiliensis Spreng. & branquilho & Av & $1,2,13,6$ & $38807,38708,38937,38224$ \\
\hline Sebastiania klotzschiana (Müll.Arg.) Müll.Arg. & branquilha & Av & $1,2,4,7,11$ & 38516 \\
\hline Sebastiania serrata (Baill.) Müll.Arg. & roxinho & Av & 4,12 & 38659,38664 \\
\hline Tetrorchidium rubrivenium Poepp. & baúna & Av & 1 & 38885,38887 \\
\hline Tragia alienata (Didr.) Múlgura \& M.M.Gut. & & $\mathrm{Li}$ & 3 & 38227 \\
\hline \multicolumn{5}{|l|}{ FABACEAE-CAESALPINIOIDEAE } \\
\hline Cassia ferruginea (Schrader) DC. & chuva-de-ouro & Av & 1,9 & 39015 \\
\hline Cassia leptophylla Vog. & falso-barbatimão & Av & 11 & \\
\hline Copaifera langsdorffii Desf.* & copaíba & Av & $1,2,5,7,14$ & $38107,38536,39233$ \\
\hline Copaifera trapezifolia Hayne & copaíba & Av & 4,2 & \\
\hline Holocalyx balansae Mich. & alecrim & Av & 12 & \\
\hline
\end{tabular}


Tabela 3. Continuação...

\begin{tabular}{|c|c|c|c|c|}
\hline Família/Espécie & Nome popular & FV & Trilha & SPSF \\
\hline Peltophorum dubium (Spreng.) Taub. & canafístula & Av & 6 & 38942 \\
\hline Senna pendula (Willdenow) Irwin \& Barneby & canudo-de-pito & Av & & 38126 \\
\hline \multicolumn{5}{|l|}{ FABACEAE-CERCIDEAE } \\
\hline Bauhinia forficata Link. & unha-de-vaca & Av & $\begin{array}{l}2,3,10,9 \\
11\end{array}$ & $38541,38901,38913$ \\
\hline Bauhinia longifolia (Bong.) Steud. & $\begin{array}{l}\text { pata-de-vaca-do- } \\
\text { campo }\end{array}$ & $\mathrm{Av}$ & $\begin{array}{l}5,7,9,11, \\
14\end{array}$ & $38573,39230,38927$ \\
\hline \multicolumn{5}{|l|}{ FABACEAE-FABOIDEAE } \\
\hline Andira anthelmia (Vell.) J.F.Macbride & garacuí & Av & 8 & 39238,38763 \\
\hline Camptosema scarlatinum (Mart. ex Benth.) Burk. & & $\mathrm{Li}$ & 7 & 38592 \\
\hline Centrolobium tomentosum Guill. ex Benth. & araribá & Av & 14 & 39229 \\
\hline Centrosema arenarium Benth. & jequitirana-da-areia & $\mathrm{Li}$ & 2 & 38591 \\
\hline Dalbergia brasiliensis Vogel & caroba-brava & Av & 2,4 & 39254 \\
\hline Dalbergia frutescens (Vell.) Britton. & assapuva & $\mathrm{Av}$ & $1,3,7$ & 39244,38925 \\
\hline Desmodium affine Schlecht. & pega-pega & Ev & 10 & 38905 \\
\hline Eriosema sp. & & $\mathrm{Li}$ & 1 & 38292 \\
\hline Erythrina crista-galli $\mathrm{L}$. & eritrina-do-banhado & Av & 8 & \\
\hline Erythrina falcata Benth. & corticeira-da-serra & $\mathrm{Av}$ & 3,12 & 38651 \\
\hline Erythrina mulungu Mart. & mulungu & Av & 10 & \\
\hline Exostyles godoyensis Soares-Silva \& Manzano & & Av & 3 & 38717,38649 \\
\hline Lonchocarpus campestris Mart. \& Benth. & embirinha & Av & $13,7,9,12$ & $38966,38967,38902$ \\
\hline Lonchocarpus cultratus (Vell.) Tozzi \& H.C.Lima & feijão-cru & Av & $1,7,11$ & 38919 \\
\hline Lonchocarpus guilleminianus (Tul.) Malme & embira-de-sapo & Av & 5,7 & 39242 \\
\hline Lonchocarpus muehlbergianus Hassler & embira-de-sapo & Av & 3,10 & 39020,38903 \\
\hline Lonchocarpus subglaucesnces Mart. ex Benth. & timbó & Av & $5,6,12$ & 38956 \\
\hline Luetzelburgia guaissara Toledo & guaiçara & Av & 5,3 & \\
\hline Machaerium aculeatum Raddi & bico-de-pato & Av & 12 & \\
\hline Machaerium acutifolium Benth. & jacarandá-do-campo & $\mathrm{Av}$ & 1 & 38090 \\
\hline Machaerium brasiliensis Vogel & sapuva & Av & $2,4,5,7$ & 39007 \\
\hline Machaerium lanceolatum (Vell.) J.F.Macbr. & & $\mathrm{Li}$ & & 38599 \\
\hline Machaerium nictitans (Vell.) Benth. & bico-de-andorinha & $\mathrm{Av}$ & $11,1,5,4$ & \\
\hline Machaerium scleroxylum Tul. & caviúna & Av & 11,1 & \\
\hline Machaerium vestitum Vogel & jacarandá-branco & Av & 7 & \\
\hline Machaerium villosum Vogel & jacarandá-do-mato & Av & 5,1 & \\
\hline Myrocarpus frondosus Allemão & cabreúva-parda & Av & 1 & \\
\hline Myroxylon peruiferum L.f. & cabreúva-vermelha & $\mathrm{Av}$ & 1 & 38645 \\
\hline Ormosia arborea (Vell.) Harms & olho-de-cabra & $\mathrm{Av}$ & 2 & \\
\hline Platypodium elegans Vogel* & amendoim-do-campo & Av & $6,7,9$ & $38571,38551,38953$ \\
\hline Pterocarpus rohrii Vahl & aldrago-miúdo & Av & $11,2,1$ & \\
\hline Rhynchosia phaseoloides (Sw.) DC. & cipó-tripa-de-galinha & $\mathrm{Li}$ & 12 & 38588 \\
\hline \multicolumn{5}{|l|}{ FABACEAE-MIMOSOIDEAE } \\
\hline Acacia paniculata Willd. & arranha-gato & Av & 5 & 38094 \\
\hline Acacia plumosa Lowe & arranha-gato & $\mathrm{Li}$ & 3,7 & 38273,38923 \\
\hline Acacia polyphylla DC. & monjoleiro & Av & 1 & \\
\hline Anadenanthera colubrina (Vell.) Brenan. & angico-branco & Av & $3,5,14$ & $38067,39234,38216$ \\
\hline Calliandra foliolosa Benth. & esponjinha & $\mathrm{Ab}$ & 1 & \\
\hline Enterolobium contortisiliquum (Vell.) Morong & tamboril & Av & 12 & \\
\hline Inga marginata Willd. & ingá-feijão & Av & $1,3,6,12$ & 38801,38970 \\
\hline Inga striata Benth. & ingá & $\mathrm{Av}$ & $\begin{array}{l}1,3,13,9, \\
12\end{array}$ & 38789,38748 \\
\hline Leucochloron incuriale (Vell.) Barneby \& J.W.Grimes* & angico-rajado & $\mathrm{Av}$ & 1 & \\
\hline
\end{tabular}


Cielo-Filho, R. et al.

Tabela 3. Continuação...

\begin{tabular}{|c|c|c|c|c|}
\hline Família/Espécie & Nome popular & FV & Trilha & SPSF \\
\hline Mimosa daleoides Benth. & mimosa & $\mathrm{Ab}$ & & 38549 \\
\hline Parapiptadenia rigida (Benth.) Brenan & angico-da-mata & Av & 1,7 & $38577,39014,39243$ \\
\hline Piptadenia gonoacantha (Mart.) J.F.Macbr. & pau-jacaré & Av & 1 & 38673 \\
\hline \multicolumn{5}{|l|}{ GESNERIACEAE } \\
\hline Sinningia allagophylla (Mart.) Wiehler* & batata-de-perdiz & Ev & & 39013 \\
\hline \multicolumn{5}{|l|}{ HYPERICACEAE } \\
\hline Hypericum brasiliense Choisy & milfurada & Ev & 9 & 38728 \\
\hline \multicolumn{5}{|l|}{ LACISTEMATACEAE } \\
\hline Lacistema hasslerianum Chodat & cafezinho & Av & $1,2,3$ & $\begin{array}{l}38253,38250,38264,38625 \\
38882,38918\end{array}$ \\
\hline \multicolumn{5}{|l|}{ LAMIACEAE } \\
\hline Aegiphila integrifolia (Jacq.) Moldenke & tamanqueira & Av & 14 & 39221 \\
\hline Aegiphila sellowiana Cham. & tamanqueiro & Av & 7,14 & 39222 \\
\hline Hyptis marrubioides Epling & hortelã-do-campo & Ev & & 38141 \\
\hline Marsypianthes chamaedrys (Vell.) Kuntze & & Ev & 3 & 38127 \\
\hline Vitex megapotamica (Spreng.) Moldenke & azeitona-do-mato & Av & 4,6 & 39028 \\
\hline Vitex montevidensis Cham. & azeitona-do-mato & Av & 4 & \\
\hline \multicolumn{5}{|l|}{ LAURACEAE } \\
\hline Cinnamomum sellowianum (Nees \& Mart.) Kosterm. & canela & Av & 2 & \\
\hline Cryptocarya aschersoniana $\mathrm{Mez}$ & canela-batalha & $\mathrm{Av}$ & $\begin{array}{l}4,3,2,5,1, \\
11\end{array}$ & \\
\hline Endlicheria paniculata (Spreng.) J.F.Macbr. & canela-do-brejo & Av & $1,2,3,9$ & $38248,38638,38989$ \\
\hline Nectandra grandiflora Nees & canelão & Av & $1,2,5,7$ & 38256,38569 \\
\hline Nectandra lanceolata Nees & canelão-amarelo & Av & $\begin{array}{l}1,3,4,13, \\
10 \\
11\end{array}$ & $\begin{array}{l}38259,38737,38747, \\
39001, \\
39019,38990\end{array}$ \\
\hline Nectandra megapotamica (Spreng.) Mez & canelinha & Av & 1,11 & 38609,38670 \\
\hline Nectandra oppositifolia Nees & canela-ferrugem & Av & 1 & \\
\hline Ocotea acutifolia $\mathrm{Mez}$ & $\begin{array}{l}\text { louro-branco-do- } \\
\text { paraná }\end{array}$ & $\mathrm{Av}$ & 5,1 & \\
\hline Ocotea catharinensis $\mathrm{Mez}$ & canela-preta & Av & 5 & \\
\hline Ocotea corymbosa $\mathrm{Mez}^{*}$ & canelinha-do-cerrado & Av & $1,2,3,4,5$ & 38995 \\
\hline Ocotea nutans (Nees) Mez & canelinha & Av & 4 & \\
\hline Ocotea puberula (Rich.) Nees & canela-gosmenta & Av & 1,12 & 38610 \\
\hline Ocotea pulchella (Nees) Mez & canela-do-cerrado & Av & 2,7 & $38257,38249,38263,39031$ \\
\hline Ocotea silvestris Vattimo-Gil & canela & Av & 2,1 & \\
\hline Ocotea $\mathrm{sp}$ & canela & Av & 1 & \\
\hline Ocotea velutina (Nees) Rohwer* & canelão & Av & $1,3,5,7,9$ & 38077,38072 \\
\hline Persea willdenovii Kosterm. & abacateiro-do-mato & Av & 1,5 & \\
\hline \multicolumn{5}{|l|}{ LAXMANNIACEAE } \\
\hline Cordyline spectabilis Kunth \& Bouché & dracena & Av & $\begin{array}{l}1,2,3,13, \\
14\end{array}$ & $38202,38198,38713,38891$ \\
\hline \multicolumn{5}{|l|}{ LECYTHIDACEAE } \\
\hline Cariniana estrellensis (Raddi) Kuntze & jequitibá-branco & Av & 12 & \\
\hline \multicolumn{5}{|l|}{ LOGANIACEAE } \\
\hline Strychnos brasiliensis (Spreng.) Mart & salta-martim & $\mathrm{Li}$ & 2,3 & 38247,38110 \\
\hline \multicolumn{5}{|l|}{ LORANTHACEAE } \\
\hline \multicolumn{5}{|l|}{ LYTHRACEAE } \\
\hline $\begin{array}{l}\text { Cuphea calophylla Cham. \& Schltdl. ssp. } \\
\text { mesostemon (Koehne) Lourteig }\end{array}$ & erva-de-bicho & Ev & 4 & 38254 \\
\hline
\end{tabular}


Tabela 3. Continuação...

\begin{tabular}{|c|c|c|c|c|}
\hline Família/Espécie & Nome popular & FV & Trilha & SPSF \\
\hline Heimia myrtifolia Cham. \& Schltdl. & quebra-arado & Ev & 10 & 39017 \\
\hline Lafoensia pacari A.St.-Hil. & dedaleiro & Av & $1,4,13,7$ & 38794,38961 \\
\hline \multicolumn{5}{|l|}{ MAGNOLIACEAE } \\
\hline Magnolia ovata A.St.-Hil. Spreng. & magnólia-do-brejo & Av & 12,14 & \\
\hline \multicolumn{5}{|l|}{ MALPIGHIACEAE } \\
\hline Banisteriopsis argyrophylla (A.Juss.) B.Gates & & $\mathrm{Li}$ & 5 & 38095 \\
\hline Bunchosia fluminensis Griseb. & ciriguela-de-padre & Av & 3,2 & \\
\hline Byrsonima intermedia A.Juss. & murici & $\mathrm{Ab}$ & 8,14 & $39196,39223,38915$ \\
\hline Diplopterys lutea (Griseb.) W.R.Anderson \& C.C.Davis & & $\mathrm{Li}$ & 13 & 38712 \\
\hline Heteropterys dumetorum (Griseb.) Nied. & & $\mathrm{Li}$ & 7 & 38804,38741 \\
\hline Heteropterys intermedia (A.Juss.) Griseb. & cipó-amarelo & $\mathrm{Li}$ & 8,14 & 39025 \\
\hline Janusia guaranitica (A.St.-Hil.) A.Juss. & & $\mathrm{Li}$ & 6 & 38947 \\
\hline Niedenzuella acutifolia (Cav.) W.R.Anderson & & $\mathrm{Li}$ & 4 & 38590,38078 \\
\hline \multicolumn{5}{|l|}{ MALVACEAE } \\
\hline Ceiba speciosa (A.St.-Hil.) Ravena & paineira & Av & & 39241 \\
\hline Gaya dominguensis Urb. & & Ev & 7 & 39030 \\
\hline Guazuma ulmifolia Lam. & mutambo & $\mathrm{Av}$ & $1,13,12$ & 38788 \\
\hline Helicteres brevispira A.St.-Hil. & sacarolha & $\mathrm{Ab}$ & 3,7 & 38759,38960 \\
\hline Luehea candicans Mart. \& Zucc. & açoita-cavalo & Av & & 39256,39253 \\
\hline Luehea divaricata Mart. & açoita-cavalo-miúdo & Av & $1,3,4,7$ & $38403,38398,38395,38964$ \\
\hline Luehea grandiflora Mart. & açoita-cavalo-graúdo & $\mathrm{Av}$ & 1,2 & $38084,38512,38545$ \\
\hline Pavonia communis A.St.-Hil. & arranca-estrepe & $\mathrm{Ab}$ & $3,6,7$ & $38255,39010,39008,39010$ \\
\hline Pavonia dusenii Krapov. & & Ev & 6 & 39027 \\
\hline Pavonia nemoralis A.St.-Hil. \& Naud. & carrapicho & Ev & 14 & 39203 \\
\hline Triumfetta semitriloba Jacq. & carrapicho & Ev & & 38136 \\
\hline Wissadula parviflora (A.St.-Hil.) R.E.Fries & malva-amarela & Ev & 5 & 38130 \\
\hline \multicolumn{5}{|l|}{ MELASTOMATACEAE } \\
\hline Acisanthera alsinaefolia Triana* & quaresmeira-do-brejo & $\mathrm{Ab}$ & 2 & 38266 \\
\hline Leandra australis (Cham.) Cogn. & & $\mathrm{Ab}$ & 1 & 38277,38268 \\
\hline Leandra melastomatoides Raddi & & Av & 2,5 & 38287,39250 \\
\hline Miconia albicans (Sw.) Triana & quaresmeira-branca & Av & $1,2,12$ & $38546,38683,38731$ \\
\hline Miconia chamissois Naudin & folha-de-bolo & Av & 4 & 38658 \\
\hline Miconia langsdorffii Cogn. & jacatirão & $\mathrm{Av}$ & 2,7 & 38290, 39029 \\
\hline Miconia ligustroides (DC.) Naudin & jacatirão-do-brejo & $\mathrm{Av}$ & $1,2,4,7,8$ & $\begin{array}{l}38083,38275,38271,38975 \\
39259\end{array}$ \\
\hline Miconia petropolitana Cogn. & jacatirão-mirim & $\mathrm{Av}$ & 2 & 38276 \\
\hline Miconia pusilliflora (DC.) Naudin & pixirica & Av & $1,2,4$ & $38274,38285,38562,38561$ \\
\hline Miconia theazans (Bonpl.) Cogn. & jacatirão-branco & $\mathrm{Ab}$ & 2 & \\
\hline Miconia tristis Spring & jacatirão-pequeno & $\mathrm{Av}$ & 3 & 38270 \\
\hline Microlepis oleaefolia (DC.) Triana & & $\mathrm{Ab}$ & 4 & 38088,38124 \\
\hline Tibouchina stenocarpa Cogn. & quaresmeira & $\mathrm{Av}$ & 2 & 38540 \\
\hline \multicolumn{5}{|l|}{ MELIACEAE } \\
\hline Cabralea canjerana (Vell.) Mart. ssp. canjerana & canjarana & Av & 1 & 38800 \\
\hline Cedrela fissilis Vell. & cedro & Av & 3,4 & 38106,38796 \\
\hline Guarea kunthiana A.Juss. & marinheiro & Av & 7,14 & 38973, 39206 \\
\hline Guarea macrophylla Vahl ssp. tuberculata (Vell.) Penn. & marinheiro & $\mathrm{Av}$ & 3,7 & 38578 \\
\hline Trichilia catigua A.Juss. & catiguá & Av & $1,4,7,11$ & $38269,38080,38994$ \\
\hline Trichilia clausseni C.DC. & catigua-de-três-folhas & $\mathrm{Av}$ & $1,3,6,14$ & $38971,38889,39213$ \\
\hline Trichilia elegans A.Juss. ssp. elegans & catiguazinho & Av & 1,3 & $38278,38284,38267,38436$ \\
\hline Trichilia pallida $\mathrm{Sw}$. & catiguá & Av & 1,11 & 38272,38511 \\
\hline
\end{tabular}


Cielo-Filho, R. et al.

Tabela 3. Continuação...

\begin{tabular}{|c|c|c|c|c|}
\hline Família/Espécie & Nome popular & FV & Trilha & SPSF \\
\hline \multicolumn{5}{|l|}{ MENISPERMACEAE } \\
\hline Abuta selloana Eichler & baga-de-caboclo & $\mathrm{Li}$ & 2,4 & 38279,38286 \\
\hline \multicolumn{5}{|l|}{ MENYANTHACEAE } \\
\hline Nymphoides indica (L.) Kuntze & estrela-branca & $\mathrm{Ev}$ & 8 & 39235 \\
\hline \multicolumn{5}{|l|}{ MONIMIACEAE } \\
\hline Mollinedia cf. elegans Tul. & pimentinha & Av & 1 & \\
\hline Mollinedia micrantha Perkins & pimentinha & Av & $1,2,5$ & $38280,38288,38890$ \\
\hline Mollinedia widgrenii A.DC. & corticeira & Av & $3,13,14$ & 38647,38714 \\
\hline \multicolumn{5}{|l|}{ MORACEAE } \\
\hline Ficus insipida Willd. & figueira & Av & 7 & 38972 \\
\hline Ficus luschnathiana (Miq.) Miq. & figueira-vermelha & Av & $2,3,7,12$ & 38220,38509 \\
\hline Ficus organensis (Miq.) Miq. & figueira-branca & Av & 1,4 & 38656 \\
\hline Ficus pulchella Schott & figueira & Av & 14 & 39211 \\
\hline Maclura tinctoria (L.) D.Don. ex Steud & taiúva & Av & 14 & 39216 \\
\hline $\begin{array}{l}\text { Sorocea bonplandii (Baill.) W.C.Burger, } \\
\text { Lanj. \& Wess.Boer }\end{array}$ & canxim & $\mathrm{Av}$ & 2,4 & $38222,38217,38619$ \\
\hline \multicolumn{5}{|l|}{ MYRSINACEAE } \\
\hline Cybianthus densicomus Mart. & & Av & 4,14 & 38623,39208 \\
\hline Rapanea ferruginea $\mathrm{Mez}$ & capororoca & Av & 6 & 38949 \\
\hline Rapanea lancifolia (Mart. ex A.DC.) Mez & capororoca & Av & 4,2 & \\
\hline Rapanea loefgrenii $\mathrm{Mez}$ & pororoca & Av & 1 & $38518,38892,38640$ \\
\hline Rapanea umbellata (Mart.) Mez & capororoca & Av & 1,2 & $\begin{array}{l}38289,38458,38445,38519, \\
38682\end{array}$ \\
\hline \multicolumn{5}{|l|}{ MYRTACEAE } \\
\hline $\begin{array}{l}\text { Blepharocalyx salicifolius } \\
\text { (Humb., Bonpl. \& Kunth) O. Berg }\end{array}$ & murta/murta-brasileira & Av & 4,1 & \\
\hline Calyptranthes concinna DC. & guamirim-facho & $\mathrm{Av}$ & $1,2,4,13,7$ & $\begin{array}{l}38734,38425,38434,38426, \\
38430,38421,38637,38755, \\
38996\end{array}$ \\
\hline Campomanesia adamantium (Camb.) O. Berg* & gabiroba-do-campo & Av & & 38724 \\
\hline Campomanesia guaviroba (DC) Kiaersk* & gabiroba & Av & $2,4,7,11$ & 39005,39022 \\
\hline Campomanesia guazumifolia (Cambess.) O. Berg & sete-capotes & Av & $1,4,7,12$ & $39246,39006,39023$ \\
\hline Campomanesia pubescens (DC.) O.Berg* & gabiroba-do-campo & Av & & 38985,39021 \\
\hline Campomanesia xanthocarpa O. Berg* & gabiroba-do-mato & Av & $1,10,7$ & $38806,38754,38984$ \\
\hline Eugenia bimarginata DC.* & falsa-cagaita & Av & 4,12 & 38635 \\
\hline Eugenia florida DC. & guamirim-cereja & Av & 14 & \\
\hline Eugenia hiemalis Camb. & cambuí-do-campo & Av & $2,3,4,5,13$ & $38808,38087,38068$ \\
\hline Eugenia involucrata DC. & cerejereira & Av & 13 & 38711 \\
\hline Eugenia ligustrina Kiaersk. & eugenia & Av & $1,2,4$ & $\begin{array}{l}38424,38435,38672,38636, \\
38987,38671\end{array}$ \\
\hline Eugenia neoverrucosa Sobral & & Av & 1 & \\
\hline Eugenia pluriflora DC. & eugenia & Av & 1 & \\
\hline Eugenia punicifolia (Humb. Bonpl. Bkunth) DC.* & cereja-do-cerrado & Av & & 39004 \\
\hline Eugenia ramboi D. Legrand & batinga-branca & Av & 12,1 & \\
\hline Eugenia stenophylla O.Berg & & Av & 1,2 & 38429,38423 \\
\hline Eugenia uniflora L.* & pitanga & Av & 3,4 & 38612 \\
\hline Gomidesia affinis (Cambess.) D.Legrand & aperta-goela & Av & $1,2,4,7$ & $38613,38422,38431$ \\
\hline Hexachlamys edulis (O.Berg) Kausel. \& D. Legrand & uvaia & Av & 1,7 & $38756,38420,38723,38611$ \\
\hline Myrceugenia sp. & guamirim & Av & 4 & \\
\hline Myrcia fallax (Rich.) DC. & $\begin{array}{l}\text { guamirim-de-folha- } \\
\text { fina }\end{array}$ & $\mathrm{Av}$ & 1,5 & $39251,5,1$ \\
\hline
\end{tabular}


Tabela 3. Continuação...

\begin{tabular}{|c|c|c|c|c|}
\hline Família/Espécie & Nome popular & FV & Trilha & SPSF \\
\hline Myrcia laruotteana Cambess. & cambuí & Av & & 38986 \\
\hline Myrcia multiflora (Lam.) DC. & cambuí & Av & $1,2,4,6$ & $38432,38805,39011,39026$ \\
\hline Myrcia pulchra Kiaersk. & & Av & $2,5,1,3$ & \\
\hline Myrcia rostrata DC. & $\begin{array}{l}\text { guamirim-de- } \\
\text { folha-fina }\end{array}$ & $\mathrm{Av}$ & $2,3,4,5,11$ & 38437,38427 \\
\hline Myrcia tomentosa (Aubl.) DC. & goiabeira-brava & Av & $5,1,2$ & \\
\hline Myrcia venulosa DC. & & $\mathrm{Av}$ & $1,2,4$ & 38438 \\
\hline Myrcianthes gigantea (D. Legrand) D. Legrand & araçá-do-mato & $\mathrm{Av}$ & 1,4 & \\
\hline Myrcianthes pungens (O.Berg.) D. Legrand & guabiju & $\mathrm{Av}$ & 1 & \\
\hline Myrciaria ciliolata Camb. & cambuí & $\mathrm{Av}$ & 4,1 & \\
\hline Myrciaria delicatula (DC.) O.Berg. & cambuí & Av & 2,7 & 38733,39272 \\
\hline Myrciaria floribunda (West. ex Willd) O. Berg. & cambuí & $\mathrm{Av}$ & $\begin{array}{l}1,2,7,12, \\
14\end{array}$ & 39024,39214 \\
\hline Myrciaria tenella (DC.) O.Berg. & cambuí & Av & 1 & \\
\hline Neomitranthes glomerata (D. Legrand) D. Legrand & guamirim-ferro & $\mathrm{Av}$ & 1,2 & 38428 \\
\hline Psidium guineense $\mathrm{S} . \mathrm{W}$. & araçá & Av & & 38738 \\
\hline Psidium rufum DC. & araçá-roxo & Av & 1,14 & \\
\hline \multicolumn{5}{|l|}{ NYCTAGINACEAE } \\
\hline Guapira hirsuta (Choisy) Lundell & maria-mole & Av & $2,3,7$ & 38455,38797 \\
\hline Guapira opposita (Vell.) Reitz & maria-mole & Av & 1 & \\
\hline \multicolumn{5}{|l|}{ OCHNACEAE } \\
\hline Ouratea spectabilis Engl.* & batiputá & Av & & 38730 \\
\hline \multicolumn{5}{|l|}{ OLEACEAE } \\
\hline Chionanthus filiformis (Vell.) P.S.Green & carne-de-vaca & Av & 3 & \\
\hline \multicolumn{5}{|l|}{ ONAGRACEAE } \\
\hline Ludwigia longifolia (DC.) H.Hara & cruz-de-malta & $\mathrm{Ab}$ & 8 & 38601 \\
\hline \multicolumn{5}{|l|}{ ORCHIDACEAE } \\
\hline Campylocentrum burchelli Cogn. & & Ep & 1 & 39805 \\
\hline Cattleya loddigesii Lindl. & cattleya & Ep & 2 & 38245 \\
\hline Liparis nervosa (Thunb.) Lindl. & & Ep & 4 & 38234 \\
\hline Oeceoclades maculata (Lindl.) Lindl. & eulofia & Ep & 5 & 38117 \\
\hline Polystachya cf. micrantha Schltr. & & Ep & 14 & 39210 \\
\hline Sacoila lanceolata (Aubl.) Garay & & Ep & 7 & 38735 \\
\hline Sauroglossum nitidum (Vell.) Schltr. & & Ep & 4 & 38662 \\
\hline \multicolumn{5}{|l|}{ PASSIFLORACEAE } \\
\hline Passiflora capsulares L. & $\begin{array}{l}\text { maracujá-branco- } \\
\text { miúdo }\end{array}$ & $\mathrm{Li}$ & 5 & 39264 \\
\hline Passiflora miersii (Mart.) Mast. & maracujazinho & $\mathrm{Li}$ & 6 & 39777 \\
\hline \multicolumn{5}{|l|}{ PERACEAE } \\
\hline Pera glabrata (Schott) Poepp. ex Baill & sapateiro & Av & 1,14 & 39224 \\
\hline \multicolumn{5}{|l|}{ PHYLLANTHACEAE } \\
\hline Hyeronima alchorneoides Allemão & sangue-de-boi & Av & 14 & 39209 \\
\hline Savia dictyocarpa Müll. Arg. & guaraiuva & Av & 1 & 38668 \\
\hline \multicolumn{5}{|l|}{ PHYTOLACCACEAE } \\
\hline Seguieria floribunda Benth. & espinho-de-juvu & $\mathrm{Av}$ & 1,12 & \\
\hline \multicolumn{5}{|l|}{ PICRAMNIACEAE } \\
\hline Picramnia parvifolia Engler & pau-amargo & Av & 6 & 38951,38934 \\
\hline Picramnia sellowii Planch. & & Av & $11,1,5$ & \\
\hline \multicolumn{5}{|l|}{ PIPERACEAE } \\
\hline Peperomia nummuralifolia (Sw.) Kunth & salva-vidas & Ep & 12 & \\
\hline Peperomia serpens (Sw.) Loudon & & Ep & 1 & 38452 \\
\hline
\end{tabular}


Cielo-Filho, R. et al.

Tabela 3. Continuação...

\begin{tabular}{|c|c|c|c|c|}
\hline Família/Espécie & Nome popular & FV & Trilha & SPSF \\
\hline Peperomia tetraphylla (G.Forst.) Hook. \& Arn. & erva-de-vidro & Ep & 3,4 & 38079,38092 \\
\hline Piper abutoloides Kunth & caapeba & $\mathrm{Ab}$ & 3 & 38648 \\
\hline Piper amalago $\mathrm{L}$. & falso-jaborandi & $\mathrm{Ab}$ & 1 & \\
\hline Piper amplum Kunth & & $\mathrm{Ab}$ & 1,13 & 38261,38710 \\
\hline Piper arboreum Aubl. & falso-jaborandi & $\mathrm{Av}$ & 1 & \\
\hline Piper corcovadensis (Miq.) C. DC. & jaguarandi & $\mathrm{Ab}$ & 1,2 & $38745,38251,38883$ \\
\hline Piper gaudichaudianum Kunth & & $\mathrm{Ab}$ & $1,2,3$ & $38262,38557,38556,38629$ \\
\hline Piper regnelli (Miq.) C. DC. & & $\mathrm{Ab}$ & 2,13 & $38252,38558,38791$ \\
\hline \multicolumn{5}{|l|}{ POACEAE } \\
\hline Lasiacis divaricata (L.) Hitchc. & taquari & $\mathrm{Ev}$ & 5 & 39247 \\
\hline Lasiacis sorghoidea (Ham.) Hitchc. \& Chase & cana-de-passarinho & Ev & 4 & 38131 \\
\hline Merostachys abadiana $\mathrm{T}$. Send. & taquara & Ev & 6,7 & 38726,38955 \\
\hline Parodiolyra micrantha (Kunth) Davidse \& Zuloaga & & Ev & 1,2 & 38639,38456 \\
\hline Paspalum paniculatum $\mathrm{L}$. & grama-touceira & $\mathrm{Ev}$ & 1 & 38897 \\
\hline \multicolumn{5}{|l|}{ POLYGALACEAE } \\
\hline Polygala fimbriata A.W.Benn. & & Ev & $4,7,9$ & $\begin{array}{l}38933,38448,38099,38922, \\
38997\end{array}$ \\
\hline Polygala lancifolia A.St.-Hil. \& Mogea & poaia-de-são-paulo & Ev & 4,5 & 38140,38096 \\
\hline \multicolumn{5}{|l|}{ POLYGONACEAE } \\
\hline Coccoloba cordata Cham. & cauaçu & Av & 2,4 & 38105 \\
\hline \multicolumn{5}{|l|}{ POLYPODIACEAE } \\
\hline Pleopeltis astrolepis (Liebm.) E.Fourn. & & Ep & 2 & 38450 \\
\hline Pleopeltis pleopeltifolia (Raddi) Alston & & Ep & 1 & 38453,38630 \\
\hline Polypodium hirsutissimum Raddi & $\begin{array}{l}\text { polipódio-hirsutíssi- } \\
\text { mo }\end{array}$ & Ep & 4 & 38101 \\
\hline \multicolumn{5}{|l|}{ PONTEDERIACEAE } \\
\hline Pontederia cordata L. & mureré & $\mathrm{Ev}$ & 8 & 38525 \\
\hline \multicolumn{5}{|l|}{ PORTULACACEAE } \\
\hline Talinum paniculatum (Jacq.) Gaertn. & joão-gomes & $\mathrm{Ev}$ & 10 & 38906 \\
\hline \multicolumn{5}{|l|}{ PROTEACEAE } \\
\hline Roupala brasiliensis Klotzsch & $\begin{array}{l}\text { carne-de-vaca-da- } \\
\text { mata }\end{array}$ & Av & $1,2,5,10,7$ & $38102,38260,38912,38762$ \\
\hline \multicolumn{5}{|l|}{ PTERIDACEAE } \\
\hline Adiantopsis radiata $(\mathrm{L}$.$) Fée$ & auacury & Ev & 1 & 38680 \\
\hline Adiantum pentadactylon Langsd. \& Fisch. & avenca-paulista & Ev & 1 & 38624 \\
\hline \multicolumn{5}{|l|}{ RANUNCULACEAE } \\
\hline Clematis dioica $\mathrm{L}$. & cipó-cruz & $\mathrm{Li}$ & 4 & 38100 \\
\hline \multicolumn{5}{|l|}{ RHAMNACEAE } \\
\hline Colubrina glandulosa Perkins & saguaragi-vermelho & Av & 4 & \\
\hline Rhamnidium elaeocarpum Reissek & saguaragi-amarelo & $\mathrm{Av}$ & 1 & \\
\hline Rhamnus sphaerosperma $\mathrm{Sw}$. & canjica & Av & 1,4 & 38093 \\
\hline \multicolumn{5}{|l|}{ ROSACEAE } \\
\hline Prunus myrtifolia (L.) Urb. & pessegueiro-bravo & $\mathrm{Av}$ & $1,5,10,7$ & $38446,38531,38506,38522$ \\
\hline Rubus brasiliensis Mart. & amora-branca & $\mathrm{Ab}$ & 2,6 & 38451,38948 \\
\hline Rubus urticifolius Poir. & nhambuí & $\mathrm{Li}$ & 4 & 38669 \\
\hline \multicolumn{5}{|l|}{ RUBIACEAE } \\
\hline Alibertia concolor (Cham.) K.Schum & marmelada & Av & $1,4,5,7,14$ & $\begin{array}{l}38244,38108,38120,38507, \\
39232\end{array}$ \\
\hline Amaioua intermedia Mart. & café-do-mato & Av & 2 & \\
\hline Chiococca alba (L.) Hitchc. & cipó-cruz & $\mathrm{Li}$ & 1 & 38633, 39239 \\
\hline Coccocypselum cordifolium Nees \& Mart.* & piririca & $\mathrm{Ev}$ & 4 & 38071 \\
\hline
\end{tabular}


Tabela 3. Continuação...

\begin{tabular}{|c|c|c|c|c|}
\hline Família/Espécie & Nome popular & FV & Trilha & SPSF \\
\hline Coccocypselum lanceolatum (Ruiz \& Pav.) Pers. & piririca & Ev & $2,4,5$ & $38240,38085,38237$ \\
\hline Coussarea contracta M.Arg. & pimenteira & Av & 3,1 & \\
\hline Emmeorhiza umbellata (Spreng.) K.Schum. & & $\mathrm{Li}$ & 2 & 38230 \\
\hline Faramea montevidensis (Cham. \& Schltdl.) DC. & carvoeiro & $\mathrm{Av}$ & 2,4 & 38228,38241 \\
\hline Galianthe brasiliensis (Spreng.) E.L.Cabral \& Bacigalupo & vassoura-de-pelote & Ev & 8 & 38760 \\
\hline Guettarda pohliana Müll.Arg. & jangada & $\mathrm{Av}$ & 12 & \\
\hline Guettarda uruguensis Cham \& Schltdl. & veludinha & $\mathrm{Ab}$ & 7 & 38962 \\
\hline Ixora venulosa Benth. & ixora & $\mathrm{Av}$ & $1,3,14$ & $38233,38238,39212$ \\
\hline Manettia cordifolia Mart. & cipó-de-santo-antônio & $\mathrm{Li}$ & 6 & 38978,39776 \\
\hline Manettia gracilis Cham. \& Schltdl. & cipó-de-santo-antônio & $\mathrm{Li}$ & 2 & 38520 \\
\hline Manettia luteo-rubra (Vell.) Benth. & fruta-de-papagaio & $\mathrm{Li}$ & 4 & 38133 \\
\hline Posoqueria latifolia (Rudge) Schult. & laranja-de-macaco & $\mathrm{Av}$ & 1,2 & 38242 \\
\hline Psychotria birotula Downs & cafezinho & $\mathrm{Ab}$ & 2,4 & $38104,38565,38567$ \\
\hline Psychotria brevicollis Müll.Arg. & & $\mathrm{Av}$ & $1,3,5$ & 38229,38134 \\
\hline Psychotria carthagenesis Jacq. & erva-de-gralha & $\mathrm{Ab}$ & 7 & 39012 \\
\hline Psychotria cephalantha (Müll.Arg.) C.M.Taylor & erva-de-rato & $\mathrm{Av}$ & 2 & 38459 \\
\hline Psychotria cf. longipes Müll.Arg. & & Av & 1 & \\
\hline $\begin{array}{l}\text { Psychotria hoffmannseggiana } \\
\text { (Willd. ex Roem. \& Schult.) Müll.Arg. }\end{array}$ & capa-rosa & $\mathrm{Ab}$ & 3,7 & 38235,38977 \\
\hline Psychotria leiocarpa Cham. \& Schltdl. & grandiúva-d'anta & $\mathrm{Ab}$ & $1,2,4$ & $38559,38089,38236,38232$ \\
\hline Psychotria velloziana Benth. & $\begin{array}{l}\text { erva-de-rato-de-folha- } \\
\text { estreita }\end{array}$ & $\mathrm{Av}$ & $1,3,4,5$ & $\begin{array}{l}38231,38070,38135,38239, \\
38246,38729\end{array}$ \\
\hline Randia armata (Sw.) DC. & fruta-de-jacaré & Av & 1,3 & \\
\hline Randia sp. & limoeiro-do-mato & Av & $1,3,10$ & 38617,38914 \\
\hline Rudgea jasminoides (Cham.) Müll.Arg. & rudgea & $\mathrm{Av}$ & $\begin{array}{l}1,2,5,10, \\
11\end{array}$ & 39248,38679 \\
\hline Simira sp. & & $\mathrm{Av}$ & 2,1 & \\
\hline \multicolumn{5}{|l|}{ RUTACEAE } \\
\hline Balfourodendron riedelianum (Engler) Engler & pau-marfim & Av & $1,12,11$ & \\
\hline Esenbeckia febrifuga (A.St.-Hil.) A.Juss. ex Mart. & mamoninha & $\mathrm{Av}$ & 2 & 38468 \\
\hline Esenbeckia grandiflora Mart. & pau-de-cotia & $\mathrm{Av}$ & $1,2,4$ & $38433,38113,38547$ \\
\hline Helietta apiculata Benth. & amarelinho & $\mathrm{Av}$ & $1,2,13,14$ & $38465,38884,39215$ \\
\hline Metrodorea nigra A.St.-Hil. & carrapateira & $\mathrm{Av}$ & $1,4,11$ & 38614,38661 \\
\hline Pilocarpus pauciflorus A.St.-Hil. & jaborandi & $\mathrm{Av}$ & $1,3,4,12$ & 38119,38627 \\
\hline Zanthoxylum fagara (L.) Sarg. & mamica-de-porca & $\mathrm{Av}$ & $\begin{array}{l}1,3,13,7, \\
12\end{array}$ & $\begin{array}{l}38529,38579,38542,38790, \\
38963\end{array}$ \\
\hline Zanthoxylum monogynum A.St.-Hil. & juvá & $\mathrm{Av}$ & 1 & \\
\hline Zanthoxylum rhoifolium Lam. & mamica-de-porca & $\mathrm{Av}$ & 3,6 & 38752,38950 \\
\hline Zanthoxylum tingoassuiba A.St.-Hil. & tinguaciba & $\mathrm{Av}$ & 1,4 & 38121 \\
\hline \multicolumn{5}{|l|}{ SALICACEAE } \\
\hline Casearia cf. aculeata Jacq. & $\begin{array}{l}\text { guaçatonga-de-espin- } \\
\text { ho }\end{array}$ & $\mathrm{Av}$ & 4 & \\
\hline Casearia decandra Jacq. & cafezeiro-do-mato & $\mathrm{Av}$ & 1,13 & 38226,38715 \\
\hline Casearia gossypiosperma Briq. & espeteiro & Av & 4 & 38655 \\
\hline Casearia lasiophylla Eichl. & cambroé & Av & 13,7 & 38792,38983 \\
\hline Casearia obliqua Spreng. & guaçatonga & $\mathrm{Av}$ & $4,3,5$ & \\
\hline Casearia sylvestris $\mathrm{Sw}$. & guaçatonga & $\mathrm{Av}$ & 1,9 & $38223,38539,38532$ \\
\hline Prockia crucis P.Browne ex L. & cuiteleiro & $\mathrm{Av}$ & 10 & 39002 \\
\hline \multicolumn{5}{|l|}{ SANTALACEAE } \\
\hline Phoradendron crassifolium (Pohl ex DC.) Eichler & $\begin{array}{l}\text { erva-de-passarinho-de- } \\
\text { folha-grande }\end{array}$ & $\mathrm{He}$ & 7 & 38574 \\
\hline
\end{tabular}


Cielo-Filho, R. et al.

Tabela 3. Continuação...

\begin{tabular}{|c|c|c|c|c|}
\hline Família/Espécie & Nome popular & FV & Trilha & SPSF \\
\hline Phoradendron mucronatum (DC.) Krug \& Urb. & erva-de-passarinho & $\mathrm{He}$ & 7 & 38595 \\
\hline Phoradendron piperoides (Kunth) Trel. & erva-de-passarinho & $\mathrm{He}$ & 3,7 & $38575,38809,38265$ \\
\hline \multicolumn{5}{|l|}{ SAPINDACEAE } \\
\hline $\begin{array}{l}\text { Allophylus edulis (A.St.-Hil., Cambess. \& A.Juss.) } \\
\text { Radlk. }\end{array}$ & três-folhas & $\mathrm{Av}$ & $\begin{array}{l}1,3,4,6,7 \\
12\end{array}$ & $38402,38758,38952,38952$ \\
\hline Cupania vernalis Cambess. & arco-de-peneira & $\mathrm{Av}$ & $4,5,13,7$ & $\begin{array}{l}38400,38066,38116,38517, \\
38743\end{array}$ \\
\hline Diatenopteryx sorbifolia Radlk. & correieira & Av & 1 & \\
\hline Matayba elaeagnoides Radlk. & cuvantã & Av & $1,2,5,7,11$ & $38399,38719,38894,38930$ \\
\hline Paullinia meliaefolia Juss. & tingui-de-folha-grande & $\mathrm{Li}$ & 5 & 38069 \\
\hline Serjania multiflora Radlk. & timpó & $\mathrm{Li}$ & 5 & 38097 \\
\hline Thinouia mucronata Radlk. & cipó-timbó & $\mathrm{Li}$ & 1 & 38281 \\
\hline Urvillea ulmacea Kunth & cipó-timbó & $\mathrm{Li}$ & $3,7,9$ & $38553,38554,38616,38626$ \\
\hline \multicolumn{5}{|l|}{ SAPOTACEAE } \\
\hline Chrysophyllum gonocarpum (Mart. \& Eichler) Engl. & guatambu-de-leite & Av & $4,13,7,12$ & 38965,38620 \\
\hline Chrysophyllum marginatum (Hook. \& Arn.) Radlk. & aguaí & Av & 5,8 & 39197 \\
\hline Pouteria gardneri (Mart. \& Miq.) Baehni & sapoti-vermelho & $\mathrm{Av}$ & 3,4 & 38469,38462 \\
\hline \multicolumn{5}{|l|}{ SCHIZAEACEAE } \\
\hline Lygodium volubile $\mathrm{Sw}$. & abre-caminho & $\mathrm{Li}$ & 1,4 & 38137,38457 \\
\hline \multicolumn{5}{|l|}{ SCHOEPFIACEAE } \\
\hline Schoepfia brasiliensis A.DC. & voadeira & $\mathrm{Av}$ & $3,1,12$ & \\
\hline \multicolumn{5}{|l|}{ SCROPHULARIACEAE } \\
\hline Buddleia stachyoides Cham. \& Schltdl. & barbasco & $\mathrm{Ab}$ & 3,7 & 38803,38666 \\
\hline \multicolumn{5}{|l|}{ SMILACACEAE } \\
\hline Smilax elastica Griseb. & japecanga & $\mathrm{Li}$ & 7 & 38924 \\
\hline Smilax quinquenervia Vell. & japecanga & $\mathrm{Li}$ & 2 & \\
\hline \multicolumn{5}{|l|}{ SOLANACEAE } \\
\hline Brunfelsia pauciflora Benth. & manacá-grado & $\mathrm{Ab}$ & 13,7 & 38926,38746 \\
\hline Cestrum bracteatum Link \& Otto & coerana & $\mathrm{Ab}$ & 1,11 & 38463,38631 \\
\hline Cestrum corymbosum Schltdl. & coerana-amarela & $\mathrm{Ab}$ & 2 & 38467 \\
\hline Cestrum laevigatum Schltdl. & coerana-branca & $\mathrm{Ab}$ & 1 & 38895 \\
\hline Cestrum mariquitense Kunth & & $\mathrm{Ab}$ & 7 & 38514 \\
\hline Cestrum schlechtendalii G.Don. & tintureiro & $\mathrm{Ab}$ & 1,3 & $38466,38470,38563$ \\
\hline Cestrum strigillatum Ruiz \& Pav. & coerana & $\mathrm{Ab}$ & 12 & 38555 \\
\hline Solanum argenteum Dunal ex Poir. & erva-de-santa-bárbara & Av & 1,11 & $38608,38642,38681$ \\
\hline Solanum concinnum Sendtn. & maria-preta-do-mato & $\mathrm{Ab}$ & 3,4 & 38461,38652 \\
\hline Solanum granulosoleprosum Scop. & fumo-bravo & $\mathrm{Ab}$ & & 38125 \\
\hline Solanum pseudocapsicum L. & peloteira & $\mathrm{Ab}$ & 6 & 38813,38943 \\
\hline Solanum sanctae-catarinae Dunal & joá-manso & $\mathrm{Ab}$ & 1,7 & 38959 \\
\hline Solanum sisymbrifolium Lam. & joá-bravo & $\mathrm{Ab}$ & 10 & 38907 \\
\hline Solanum variabile Mart. & jurubeba-velame & $\mathrm{Ab}$ & 6,7 & $38795,38939,38917$ \\
\hline \multicolumn{5}{|l|}{ STYRACACEAE } \\
\hline Styrax latifolius Pohl & canela-póca & Av & $2,5,9,8$ & $38460,38749,39258$ \\
\hline Styrax pohli A.DC. & estoraque & Av & 1,5 & \\
\hline \multicolumn{5}{|l|}{ SYMPLOCACEAE } \\
\hline Symplocos celastrinea Mart. ex. Miq. & caá-apoam & Av & 2 & \\
\hline Symplocos pubescens Klotzsch ex Benth. & sete-sangrias & Av & 3 & \\
\hline Symplocos sp. & sete-sangrias & Av & 2 & \\
\hline Symplocos variabilis Mart. ex. Miq. & congonha-grande & $\mathrm{Av}$ & 2 & 38464 \\
\hline \multicolumn{5}{|l|}{ THYMELAEACEAE } \\
\hline Daphnopsis fasciculata (Meisn.) Neul. & embira & Av & 4 & \\
\hline
\end{tabular}


Tabela 3. Continuação...

\begin{tabular}{|c|c|c|c|c|}
\hline Família/Espécie & Nome popular & FV & Trilha & SPSF \\
\hline Daphnopsis racemosa Griseb. & embira-de-brejo & $\mathrm{Av}$ & 1 & 38243 \\
\hline \multicolumn{5}{|l|}{ URTICACEAE } \\
\hline Cecropia pachystachya Trécul & embaúba & Av & & 38524 \\
\hline Coussapoa microcarpa (Schott) Rizzini & figueira & Av & 4 & \\
\hline \multicolumn{5}{|l|}{ VERBENACEAE } \\
\hline Aloysia virgata Juss. & lixeira & Av & 4,10 & 39016,38618 \\
\hline Citharexylum myrianthum Cham. & pau-viola & $\mathrm{Av}$ & & 39240 \\
\hline Lantana brasiliensis Link & cambará-branco & $\mathrm{Ab}$ & 5 & 39245 \\
\hline Lantana camara $\mathrm{L}$ & cambará-de-espinho & $\mathrm{Ab}$ & 3 & 38576,38722 \\
\hline Lantana canescens Kunth & cambarazinho & $\mathrm{Ab}$ & 4 & 38665 \\
\hline Lantana fucata Lindl. & cambará-roxo & $\mathrm{Ab}$ & 1,4 & 38401,38622 \\
\hline Petrea volubilis $\mathrm{L}$. & pétrea & $\mathrm{Li}$ & 1 & 38396,38721 \\
\hline Stachytarpheta cayanensis (Rich.) Vahl & gervão & Ev & 10 & 38991 \\
\hline Verbena rigida Spreng. & verbena & Ev & 3,10 & 38718,39000 \\
\hline \multicolumn{5}{|l|}{ VIOLACEAE } \\
\hline Hybanthus atropurpureus (A.St.-Hil.) Taub. & ganha-saia & $\mathrm{Ab}$ & 5 & \\
\hline Hybanthus bigibbosus (A.St.-Hil.) Hassl. & erva-de-veado & $\mathrm{Ab}$ & $1,3,13,10$ & $38742,38750,38628,38904$ \\
\hline \multicolumn{5}{|l|}{ VITACEAE } \\
\hline Cissus erosa Rich.* & cipó-de-fogo & $\mathrm{Li}$ & 8 & 39237 \\
\hline \multicolumn{5}{|l|}{ VOCHYSIACEAE } \\
\hline Qualea cordata Spreng.* & carvãozinho & $\mathrm{Av}$ & 2 & \\
\hline Qualea grandiflora Mart.* & pau-terra & Av & & \\
\hline Vochysia magnifica Warm. & pau-novo & $\mathrm{Av}$ & 1 & \\
\hline Vochysia tucanorum Mart. & cinzeiro & Av & $2,7,14$ & 38550,39217 \\
\hline
\end{tabular}

1998, Hora \& Soares 2002, Udulutsh et al. 2004, Rezende \& Ranga 2005, Tibiriçá et al. 2006, Rezende et al. 2007), conforme citado por Gentry (1991) para as regiões tropicais. Na área de estudo, ocorreram 72 espécies de lianas, sendo que $42 \%$ delas pertencem às famílias Bignoniaceae, Apocynaceae e Asteraceae.

As famílias Cyperaceae, Malvaceae e Poaceae reuniram o maior número de ervas. As famílias Solanaceae, Piperaceae e Asteraceae foram as mais ricas em espécies arbustivas. Conforme Stranghetti \& Ranga (1998) e Kinoshita et al. (2006), Piperaceae, Rubiaceae e Solanaceae ocorrem entre as famílias mais ricas em espécies para o hábito arbustivo. Acanthaceae, Poaceae e Asteraceae são relatadas como as de maior riqueza específica entre as herbáceas.

A família Orchidaceae foi a mais rica em espécies de epífitas, com sete espécies. As demais famílias: Bromeliaceae, Cactaceae, Piperaceae e Polypodiaceae apresentaram todas três espécies. Informações sistematizadas sobre a ocorrência de epífitas nas florestas semideciduais paulistas são escassas na literatura. Breier (2005) encontrou 25 espécies epifíticas na Floresta Estacional da Estação Ecológica de Caetetus. As famílias mais ricas reportadas pelo autor foram Polypodiaceae, Piperaceae e Cactaceae. Para um fragmento florestal em Campinas, Bernacci \& Leitão Filho (1996) comentam a ocorrência de bromeliáceas de pequeno porte, mas em número reduzido de espécies e indivíduos, em comparação a áreas onde o clima é mais úmido, e atribuem a ausência de orquidáceas à extração seletiva na área estudada. Por outro lado, Breier (2005) comenta a redução da proporção de espécies de orquidáceas e o aumento de polipodiáceas em florestas com estacionalidade climática marcada. Na área de estudo deste trabalho, a presença de ambientes mais úmidos, a proteção da área e possíveis vieses de amostragem podem estar relacionados à predominância de orquidáceas na flora epifítica.
A amostra de composição florística obtida na Floresta Estadual e Estação Ecológica de Paranapanema apresentou um número de espécies superior ao encontrado em outras amostras de flora da Floresta Estacional Semidecidual paulista, evidenciando a elevada riqueza florística das unidades de conservação do município de Paranapanema. O número de espécies de plantas vasculares registradas na área de estudo é semelhante ao relatado em estudos similares em áreas de Floresta Ombrófila Densa no estado de São Paulo (Ivanauskas et al. 2001, Ziparro et al. 2005), o que denota, de forma comparativa, a importância da Floresta Estacional Semidecidual para a conservação da biodiversidade paulista.

A percentagem de espécies ameaçadas na área de estudo é de aproximadamente $3 \%$, de acordo com as listas consultadas. O objetivo das listas de espécies ameaçadas é orientar as ações de políticas públicas voltadas à pesquisa e à proteção da biodiversidade. As listas são o primeiro passo para a conservação das espécies, pois implicam na capacidade de monitorar o status de ameaça da biodiversidade (IUCN 2001). Tais espécies recebem tutela legal da legislação ambiental brasileira (Brasil 1998) e internacional (Brasil 2000). A criação e manutenção de unidades de conservação constituem as principais medidas para a proteção de espécies e habitat ameaçados e reversão da tendência de extinção. A presença de tais espécies em uma Unidade de Conservação representa, portanto, não apenas um atestado da importância da unidade, mas também implica em grande responsabilidade para os gestores da área. Uma vez detectada a presença de espécies ameaçadas, devem ser implementadas ações visando à pesquisa e ao monitoramento, de modo a fornecer subsídios para programas de conservação.

A maior parte dos tipos de ameaça envolve o desmatamento e/ou a exploração da madeira. Tais ameaças são quase nulas em unidades 
Tabela 4. Espécies ameaçadas de extinção registradas na Floresta Estadual de Paranapanema e Estação Ecológica de Paranapanema segundo as listas da União Internacional para a Conservação da Natureza (IUCN), Fundação Biodiversitas (FB) e Secretaria do Meio Ambiente do estado de São Paulo (SMA-SP). EX, Extinta na Natureza; EN, Em Perigo; VU, vulnerável. As espécies ameaçadas de acordo com a lista do Ministério do Meio Ambiente (MMA) estão sinalizadas.

Table 4. Threatened species recorded at the State Forest of Paranapanema and Ecological Station of Paranapanema according to the lists of the International Union for Conservation of Nature (IUCN), Biodiversitas Foundation (FB) and Environmental Bureau of the São Paulo state (SMA-SP). EX, Extinct in the Wild; EN, Endangered; VU, Vulnerable. The species threatened according to the Brazilian Environmental Agency (MMA) are indicated.

\begin{tabular}{|c|c|c|c|c|c|}
\hline Família/Espécie & IUCN & FB & SMA-SP & MMA & Tipo de ameaça* \\
\hline \multicolumn{6}{|l|}{ APOCYNACEAE } \\
\hline Aspidosperma polyneuron Müll.Arg. & EN & & & & Exploração da madeira \\
\hline \multicolumn{6}{|l|}{ ARECACEAE } \\
\hline Butia archeri (Glassman) Glassman & & & EX & & \\
\hline Euterpe edulis Mart. & & EN & VU & $\operatorname{sim}$ & Exploração do palmito \\
\hline \multicolumn{6}{|l|}{ BIGNONIACEAE } \\
\hline Zeyheria tuberculosa (Vell.) Bur. & VU & & & & Desmatamento \\
\hline \multicolumn{6}{|l|}{ FABACEAE-FABOIDEAE } \\
\hline Luetzelburgia guaissara Toledo & & & VU & & Baixa densidade populacional \\
\hline Machaerium villosum Vogel & VU & & & & Exploração da madeira e desmatamento \\
\hline Myroxylum peruiferum L.f. & & & VU & & Exploração da madeira e desmatamento \\
\hline \multicolumn{6}{|l|}{ LAURACEAE } \\
\hline Ocotea catharinensis $\mathrm{Mez}$ & VU & & & $\operatorname{sim}$ & Exploração da madeira \\
\hline \multicolumn{6}{|l|}{ MALVACEAE } \\
\hline Gaya dominguensis Urb. & & & VU & & Distribuição geográfica restrita \\
\hline \multicolumn{6}{|l|}{ MELIACEAE } \\
\hline Cedrela fissilis Vell. & EN & & & & Exploração da madeira e desmatamento \\
\hline \multicolumn{6}{|l|}{ MORACEAE } \\
\hline Ficus pulchella Schott & VU & & & & Desmatamento e fragmentação de hábitat \\
\hline \multicolumn{6}{|l|}{ MYRTACEAE } \\
\hline Myrcianthes pungens (O.Berg) D. Legrand & EN & & & & Desmatamento e fragmentação de hábitat \\
\hline \multicolumn{6}{|l|}{ PIPERACEAE } \\
\hline Peperomia serpens (Sw.) Loudon & & & VU & & Distribuição geográfica restrita \\
\hline \multicolumn{6}{|l|}{ POACEAE } \\
\hline Merostachys abadiana Sendulsky & & & EN & & Distribuição geográfica restrita \\
\hline \multicolumn{6}{|l|}{ RUTACEAE } \\
\hline Baufourodendron riedelianum (Engler) Engler & EN & & & & Exploração da madeira e desmatamento \\
\hline
\end{tabular}

Fontes: www.iucnredlist.org, www.biodiversitas.org.br (Acessos em março de 2009) e Mamede et al. 2007.

de conservação com proteção efetiva. Por outro lado, a exploração de palmito, que atinge a espécie Euterpe edulis Mart., é um tipo de ameaça difícil de conter, mesmo em unidades de conservação bem protegidas, merecendo maior atenção. É preciso reconhecer, entretanto, que as populações de espécies ameaçadas dentro de unidades de conservação com proteção efetiva dependem, para se manterem viáveis em longo prazo, de trocas gênicas com outras populações coespecíficas que se encontram fora da unidade (Ghazoul 2005, Knight et al. 2005, Vamosi et al. 2006). Assim, uma população de espécie ameaçada, seja qual for o tipo de ameaça, não perde automaticamente o status de ameaçada por ocorrer em uma unidade de conservação e requer monitoramento contínuo. A efetiva conservação dessa população depende de medidas dentro e fora da unidade, que permitam a troca gênica com outras populações.

\section{Agradecimentos}

Somos gratos aos pesquisadores do Instituto Florestal Hideyo Aoki e Geraldo Antonio Daher Corrêa Franco pelo apoio logístico e auxílio na identificação de espécies, respectivamente; à Dra. Andréia
Alves Rezende, por colaborar na identificação de espécies de lianas; à pesquisadora Marina Mitsue Kanashiro e ao Raphael Ferraz, pela elaboração da figura e fornecimento dos dados correspondentes; à Betânia Monteiro Cielo, pela revisão ortográfica e gramatical do manuscrito; aos senhores Donizete e Natal, funcionários da Estação Ecológica de Paranapanema, pelo auxilio em campo, e ao técnico do Herbário Dom Bento Pickel, Ernane Lino da Silva, pelo apoio no processamento e montagem do material botânico. Este trabalho contou com a indispensável contribuição de vários taxonomistas especialistas aos quais gostaríamos de expressar nosso agradecimento especial.

\section{Referências Bibliográficas}

AGUIAR, O.T. 2004. Comparação entre métodos de quadrantes e parcelas na caracterização da composição florística e fitossocioló de um trecho de Floresta Ombrófila Densa no Parque Estadual "Carlos Botelho" São Miguel Arcanjo, SP. Dissertação de Mestrado, Escola Superior de Agricultura "Luiz de Queiroz", Universidade de São Paulo, Piracicaba, p. 119. 
AOKI, H., PASQUAL, A., ESTEVES, R. \& NAGY, S.C.S. 2001. Plano de Manejo da Estação Ecológica de Paranapanema - SP. IF Série Registros. 23:1-19.

BERNACCI, L.C. \& LEITÃO-FILHO, H.F. 1996. Flora fanerogâmica da fazenda São Vicente, Campinas, SP. Rev. bras. bot. 19(2):149-164.

BIODIVERSITAS. Lista oficial de espécies ameaçadas de extinção no Brasil. http://www.biodiversitas.org.br./florabr/grupo3fim.asp. (último acesso em 10/12/2008)

BRASIL. Decreto $n^{\circ}$ 02, de 03 de Fevereiro de 1994. Aprova o texto da Convenção sobre Diversidade Biológica, assinada durante a Conferência das Nações Unidas sobre Meio Ambiente e Desenvolvimento, realizada na cidade do Rio de Janeiro, no período de 5 a 14 de junho de 1992. Diário Oficial da União, de 03 de Fevereiro de 1994.

BRASIL. Lei n ${ }^{\circ}$ 9.605, de 12 de Fevereiro de 1998. Dispõe sobre as sanções penais e administrativas derivadas de condutas e atividades lesivas ao meio ambiente, e dá outras providências. Diário Oficial da União, de 13 de Fevereiro de 1998.

BRASIL. Decreto n 3.607 , de 21 de Setembro de 2000. Dispõe sobre a implementação da Convenção sobre Comércio Internacional das Espécies da Flora e Fauna Selvagem em Perigo de Extinção - CITES, e dá outras providências. Diário Oficial da União, de 22 de Setembro de 2000.

BREIER, T.B. 2005. O epifitismo vascular em florestas do sudeste do Brasil. Tese de Doutorado, Universidade Estadual de Campinas, Campinas, p. 139.

CASTRO, A.A.J.F., MARTINS, F.R., TAMASHIRO, J.Y. \& SHEPHERD, G.J. 1999. How rich is the flora of Brazilian cerrados? Ann. Missouri Bot. Gard. 86(1):192-224.

CIELO-FILHO, R. \& SANTIN, D. 2002. Estudo florístico e fitossociológico de um fragmento florestal urbano: Bosque dos Alemães, Campinas, SP. Rev. bras. bot. 25(3):291-301.

CUSTODIO-FILHO, A., NEGREIROS, O.C., DIAS, A.C., FRANCO, G.A.D.C. 1992. Composição florística do estrato Arbóreo do Parque Estadual de Carlos Botelho, SP. In Anais do II Congresso Nacional de Essências Nativas. Instituto Florestal, São Paulo, p. 184-191. (v. 4).

DIAS, A.C. 2004. Composição Florística, fitossociológica, diversidade de espécies arbóreas e comparação de métodos de amostragem na Floresta Ombrófila Densa do Parque Estadual Carlos Botelho - SP, Brasil. Tese de Doutorado, Escola Superior de Agricultura "Luiz de Queiroz", Universidade de São Paulo, p. 184.

DURIGAN, G., FRANCO, G.A.D.C., MASAHIRO, S. \& BAITELLO, J.B. 2000. Estrutura e diversidade do componente arbóreo da floresta na Estação Ecológica dos Caetetus, Gália, SP. Rev.bras. bot. 23(4):371-383.

DURIGAN, G., MAMEDE, M.C.H., IVANAUSKAS, N.M., SIQUEIRA, M.F., JOLY, C.A., MOURA, C., BARROS, F., SOUZA, F.M., VILELA, F.E.S.P., ARZOLLA, F.A.R.P., FRANCO, G.A.D.C., CORDEIRO, I., KOCH, I., BAITELLO, J.B., LOMBARDI, J.A., LIMA, L.R., LOHMENN, L.G., BERNACCI, L.C., ASSIS, M.A., AIDAR, M.P.M., WANDERLEY, M.G.L., TONIATO, M.T.Z., RIBEIRO, M., GROPPO, M., CAVASSAN, O., SANO, P.T., RODRIGUES, R.R., FICHS, T.V. \& MARTINS, S.E. 2008. Fanerógamas. In Diretrizes para a conservação e restauração da biodiversidade no Estado de São Paulo (R.R. Rodrigues \& V.L.R. Bononi, orgs). Instituto de Botânica/Imprensa Oficial do Estado de São Paulo, São Paulo, p. 104-109.

FIDALGO, O. \& BONONI, V.L.R. 1989. Técnicas de coleta, preservação e herborização de material botânico. Instituto de Botânica/Imprensa Oficial do Estado de São Paulo, São Paulo, p. 62. (série documentos).

FONSECA, R.C.B. \& RODRIGUES, R.R. 2000. Análise estrutural e aspectos do mosaico sucessional de uma floresta semidecídua em Botucatu, SP. Sci. for.Scientia Florestalis. 57:27-43.

GHAZOUL, J. 2005. Pollen and seed dispersal among dispersed plants. Biol. rev. 80:413-443.

GENTRY, A.H. 1991. The distribution and evolution of climbing plants. In The biology of vines (F.E. Putz \& H.A. Mooney, orgs). Cambridge University Press, Cambridge, p. 3-49.
GIULIETTI, A.M., RAYMOND, M.H., QUEIROZ, L.P. \& WANDERLEY M.G.L., van den BERG, C. 2005. Biodiversity and conservation of plants in Brazil. Conserv. biol. 19(3):632-639.

HORA, H.C. \& SOARES, J.J. 2002. Estrutura fitossociológica da comunidade de lianas em uma floresta estacional semidecidual na Fazenda Canchim, São Carlos, SP. Rev. bras. bot. 25(3):323-329.

International Plant Names Index - IPNI. http://www.ipni.org/ipni/ plantnamesearchpage.do (último acesso em 10/12/2008).

International Union for Conservation of Nature - IUCN. 2001. IUCN Red list categories and criteria. Version. 3.1. IUCN Species Survival Commission, Suíça, p. 30.

International Union for Conservation of Nature - IUCN. 2006. Lista vermelha de espécies ameaçadas de extinção da união internacional para a conservação da natureza. http://www.iucnredlist.org (último acesso em $5 / 12 / 2008)$

IVANAUSKAS, N.M., RODRIGUES, R.R. \& NAVE, A.G. 1999. Fitossociologia de um trecho de floresta estacional semidecidual no município de Itatinga, São Paulo, Brasil. Sci. for. Scientia Florestalis. 56:83-99.

IVANAUSKAS, N.M., MONTEIRO, R. \& RODRIGUES, R.R. 2001. Levantamento florístico de trecho de Floresta Atlântica em Pariquera Açu, São Paulo, Brasil. Naturalia. 26:97-129.

JUDD, W.S., CAMPBELL, C.S., KELLOG, E.A., STEVENS, P.F. \& DONOGHUE, M.J. 2002. Plant systematics: a phylogenetic approach. Sinauer Associates Inc., Sunderland, p. 576.

KINOSHITA, L.S., TORRES, R.B., FORNI-MARTINS, E.R., SPINELLI, T., AHN, Y.J. \& CONSTÂNCIO, S.B. 2006. Composição florística e síndromes de polinização e de dispersão da mata do Sítio São Francisco, Campinas, SP, Brasil. Acta bot. bras. 20(2):213-237.

KNIGHT, T.M., STEETS, J.A., VAMOSI, J.C., MAZER, S.J., BURD, M., CAMPBELL, D.R., DUDASH, M.R., JOHNSTON, M.O., MITCHELL, R.J. \& ASHMAN, T.L. 2005. Pollen limitation and plant reproduction: pattern and process. Ann. rev. ecol. evol. syst. 36:467-497.

KRONKA, F.J.N., NALON, M.A., MATSUKUMA, C.K., KANASHIRO, M.M., YWANE, M.S.S., PAVÃO, M., DURIGAN, G., LIMA, L.M.P.R., GUILLAUMON, J.R., BAITELLO, J.B. \& BARRADAS, A.M.F 2005. Inventário florestal da vegetação natural do Estado de São Paulo. Secretaria do Meio Ambiente/Instituto Florestal/Imprensa Oficial, São Paulo, p. 200.

KURY, A.B. 2006. Diretrizes e estratégias para a modernização de coleções biológicas brasileiras e a consolidação de sistemas integrados de informação sobre biodiversidade. Ministério da Ciência e Tecnologia/ Centro de Gestão e Estudos Estratégicos, Brasília, p. 314.

LEITÃO-FILHO, H.F. 1982. Aspectos taxonômicos das florestas do Estado de São Paulo. Silvic. São Paulo, p. 197-206. (v. 16).

LEITÃO-FILHO, H.F. 1987. Considerações sobre a florística de florestas tropicais e subtropicais do Brasil. Instituto de Pesquisa e Estudos Florestais, Piracicaba, p. 41-46. (v. 35).

LEWINSOHN, T.M. \& PRADO, P.I. 2002. Biodiversidade brasileira: síntese do estado atual do conhecimento. Contexto, São Paulo, p. 176.

MAMEDE, M.C.H. 2003. A experiência do projeto Flora Fanerogâmica do Estado de São Paulo. In Coleções biológicas de apoio ao inventário, uso sustentável e conservação da biodiversidade (A.L. Peixoto, ed.). Instituto de Pesquisas Jardim Botânico do Rio de Janeiro, Rio de Janeiro, p. $127-139$

MAMEDE, M.C.H., SOUZA, V.C., PRADO, J., BARROS, F., WANDERLEY, M., DAS, G.L. \& RANDO, J.G. 2007. Livro vermelho das espécies vegetais ameaçadas do Estado de São Paulo. Instituto de Botânica/ Imprensa Oficial do Estado de São Paulo, São Paulo, p. 165.

MENDONÇA, R.C., FELFILI, J.M., WALTER, B.M.T., SILVA Jr., M.C., REZENDE, A.V., FILGUEIRAS, T.S. \& NOGUEIRA, P.E. 1998. Flora vascular do cerrado. In Cerrado: ambiente e flora (S.M. Sano \& S.P. Almeida, eds). EMBRAPA/CPAC, Planaltina, p. 290-556. 
Ministério do Meio Ambiente - MMA. 2008. Instrução normativa nº 06, de 26 de setembro de 2008. http://www.mma.gov.br (último acesso em 05/12/2008).

Ministério do Meio Ambiente - MMA. 2000. Avaliação e ações prioritárias para a conservação da biodiversidade da Mata Atlântica e Campos Sulinos. DELREY, Brasília, 40 p.

Missouri Botanical Garden - MOBOT. http://www.tropicos.org/ (último acesso em 05/12/2008).

MitTERMEIER, R.A., FONSECA, G.A.B., RYLANDS, A.B. \& MITTERMEIER, C.G. 1997. Atlantic Forest. In Megadiversity: earth's biologically wealthiest nations Brazil. (R.A. Mittermeier, P. Robles Gil \& C.G. Mittermeier, eds). CEMEX, México, p. 39-49.

PEIXOTO, A.L. \& MORIM, M.P. 2003. Coleções botânicas: documentação da biodiversidade brasileira. Cien. cult., 55(3):21-24.

PEIXOTO, A.L. \& THOMAS, W.W. 2005. Listas florísticas, floras regionais e flora do Brasil: desafios e estratégias. http//:www.cria.org.br/cgee/col (último acesso em 10/01/2007).

PETERSON, A.T. 2001. Predicting species' geographic distributions based on ecological niche modeling. Condor. 103(3):599-605.

PIRANI, J.R. 2005. Sistemática: tendências e desenvolvimento, incluindo impedimentos para o avanço do conhecimento na área. http//:www.cria. org.br/cgee/col (último acesso em 10/01/2007).

PROENÇA, C.E.B., MUNHOZ, C.B.R., JORGE, C.L. \& NÓBREGA, M.G.G. 2001. Listagem e nível de proteção das espécies de fanerógamas do Distrito Federal, Brasil. In Flora do Distrito Federal, Brasil (T.B. Cavalcanti \& A.E. Ramos, orgs). EMBRAPA, Brasília, p. 89-359.

REZENDE, A.A. \& RANGA, N.T. 2005. Lianas da Estação Ecológica do Noroeste Paulista, São José do Rio Preto/Mirassol, SP. Acta bot. bras. 19(2):273-279.

REZENDE, A.A., RANGA, N.T. \& PEREIRA, R.A.S. 2007. Lianas de uma floresta estacional semidecidual, município de Paulo de Faria, Norte do Estado de São Paulo, Brasil. Rev. bras. bot. 30(3):451-461.

RODRIGUES, R.R. \& BONONI, V.L.R. 2008. Diretrizes para a conservação e restauração da biodiversidade no Estado de São Paulo. Instituto de Botânica/Imprensa Oficial do Estado de São Paulo, São Paulo, p. 238.

SANTOS, K. \& KINOSHITA, L.S. 2003. Flora arbustivo-arbórea do fragmento de Floresta Estacional Semidecidual do Ribeirão Cachoeira, município de Campinas, SP. Acta bot. bras. 17(3):325-486.

SÃO PAULO. Resolução SMA 48, de 21 de setembro de 2004. http://www. ibot.sp.gov.br (último acesso em 10/12/2008).

SCARAMUZZA, C.A.M. 2006. Flora e ecologia dos campos de Itararé, São Paulo, Brasil. Tese de Doutorado, Universidade de São Paulo, São Paulo, p. 153.

SHEPHERD, G.J. 2003. Avaliação do estado do conhecimento da diversidade biológica do Brasil: Plantas terrestres (versão preliminar). Ministério do Meio Ambiente/Secretaria de Biodiversidade e Florestas, Brasília. http//: www.mma.gov.br/estruturas/chm/_arquivos/plantas1.pdf (último acesso em 10/01/2007).

SILVA, L.A. \& SOARES, J.J. 2003. Composição florística de um fragmento de floresta estacional semidecídua no município de São Carlos - SP. Rev. árvore. 27(5):647-656.

Species Link. Disponível em: http://www.splink.cria.org.br (último acesso em 07/07/2009).
STOCKWELL, D.R.B. \& PETERSON, A.T. 2002. Effects of sample size on accuracy of species distribution models. Ecol. model. 148(1):1-13.

STRANGHETTI, V. \& RANGA, N.T. 1998. Levantamento florístico das espécies vasculares da floresta estacional mesófila semidecídua da Estação Ecológica de Paulo de Faria - SP. Rev. bras. bot. 21(3):289-298.

TIBIRIÇÁ, Y.J.A., COELHO, L.F.M. \& MOURA, L.C. 2006. Florística de lianas em um fragmento de floresta estacional semidecidual, Parque Estadual de Vassunga, Santa Rita do Passa Quatro, SP, Brasil. Acta bot. bras. 20(2):339-346.

TORRES, R.B. 1989. Estudos florísticos em mata secundária da Estação Ecológica de Angatuba, Angatuba (São Paulo). Dissertação de Mestrado, Universidade Estadual de Campinas, Campinas, p. 231.

UDULUTSH, R.G., ASSIS, M.A. \& PICCHI, D.C. 2004. Florística de trepadeiras numa floresta estacional semidecídua, Rio Claro - Araras, Estado de São Paulo, Brasil. Rev. bras. bot. 27(1):125-134.

VAMOSI, J.C., KNIGHT, T.M., STEETS, J.A., MAZER, S.J., BURD, M. \& ASHMAN, T.L. 2006. Pollination decays in biodiversity hotspots. Proc. Natl. Acad. Sci. U.S.A.103(4):956-961.

VENTURA, A., BERENGUT, G. \& VICTOR, M.A.M. 1965. Características edafo-climáticas das dependências do Serviço Florestal de São Paulo. Silvic, São Paulo, p. 57-140. (v. 4).

WANDERLEY, M.G.L., SHEPHERD, G.J. \& GIULIETTI, A.M. 2001. Flora Fanerogâmica do Estado de São Paulo. FAPESP/HUCITEC, São Paulo, p. 292. (v. 1).

WANDERLEY, M.G.L., SHEPHERD, G.J. \& GIULIETTI, A.M. 2002. Flora Fanerogâmica do Estado de São Paulo. FAPESP/HUCITEC, São Paulo, p. 391. (v. 2).

WANDERLEY, M.G.L., SHEPHERD, G.J., GIULIETTI, A.M. \& MELHEM, T.S. 2003. Flora Fanerogâmica do Estado de São Paulo. FAPESP/RIMA, São Paulo, p. 367. (v. 3).

WANDERLEY, M.G.L., SHEPHERD, G.J., MELHEM, T.S. \& GIULIETTI, A.M. 2005. Flora Fanerogâmica do Estado de São Paulo. FAPESP/RIMA, São Paulo, p. 392. (v. 4).

WANDERLEY, M.G.L., SHEPHERD, G.J., MELHEM, T.S. \& GIULIETTI, A.M. 2007. Flora Fanerogâmica do Estado de São Paulo. FAPESP, São Paulo, p. 476. (v. 5).

YAMAMOTO, L.F., KINOSHITA, L.S. \& MARTINS, F.R. 2005. Florística dos componentes arbóreo e arbustivo de um trecho de floresta estacional semidecídua montana, no município de Pedreira, Estado de São Paulo. Rev. bras. bot. 28(1):191-202.

ZIPARRO, V.B., GUILHERME, F.A.G., ALMEIDA-SCABBIA, R.J. \& MORELLATO, L.P.C. 2005. Levantamento florístico de floresta Atlântica no sul do estado de São Paulo, Parque Estadual de Intervales, Base Saibadela. Biota neotrop. 5(1):1-24. http://www.biotaneotropica. org.br/v5n1/pt/abstract?inventory+BN02605012005. (último acesso em 10/01/2007).

Recebido em 22/12/08

Versão reformulada recebida em 08/07/09

Publicado em 03/08/09 